\title{
Some combinatorial principles defined in terms of elementary submodels
}

\author{
by \\ Sakaé Fuchino (Kasugai) and Stefan Geschke (Berlin)
}

\begin{abstract}
We give an equivalent, but simpler formulation of the axiom SEP, which was introduced in [9] in order to capture some of the combinatorial behaviour of models of set theory obtained by adding Cohen reals to a model of $\mathrm{CH}$. Our formulation shows that many of the consequences of the weak Freese-Nation property of $\mathcal{P}(\omega)$ studied in [6] already follow from SEP. We show that it is consistent that SEP holds while $\mathcal{P}(\omega)$ fails to have the $\left(\aleph_{1}, \aleph_{0}\right)$-ideal property introduced in [2]. This answers a question addressed independently by Fuchino and by Kunen. We also consider some natural variants of SEP and show that certain changes in the definition of SEP do not lead to a different principle, answering a question of Blass.
\end{abstract}

1. Introduction. As far as the combinatorial properties of the reals are concerned, among the best understood models of set theory are Cohen models, i.e., models of set theory obtained by adding Cohen reals to a model of $\mathrm{CH}$. In order to get rid of the technical and meta-mathematical difficulties of forcing, it is worthwhile to isolate the combinatorial features of Cohen models in the form of easily applicable combinatorial principles.

This has been done by various authors. The work of Juhász, Szentmiklóssy, and Soukup [10,11] can be considered as the starting point of this line of research. The intention of the present article is to clarify the interrelations between three similar principles that turned up in this context, namely SEP introduced by Juhász and Kunen [9], the $\left(\aleph_{1}, \aleph_{0}\right)$-ideal property (IDP for short) of Dow and Hart [2] and $\operatorname{WFN}(\mathcal{P}(\omega))$ from [6].

SEP and IDP are originally defined by the same pattern. The principles say that for every sufficiently large regular cardinal $\chi$ there are many good

2000 Mathematics Subject Classification: Primary 03E35; Secondary 03E05, 03E17, $03 \mathrm{E} 65$.

Key words and phrases: weak Freese-Nation property, SEP, Cohen model, almost disjoint number.

The first author thanks Hiroshi Sakai for pointing out a gap in the proof of an earlier version of Theorem 8.14. 
elementary submodels $M$ of the structure $\left(\mathcal{H}_{\chi}, \in\right)$ such that $\mathcal{P}(\omega) \cap M$ is nicely embedded in $\mathcal{P}(\omega)$. Here $\mathcal{H}_{\chi}$ denotes the family of all sets whose transitive closure is of size $<\chi$. Structures of the form $\left(\mathcal{H}_{\chi}, \in\right)$ satisfy a large part of ZFC.

Different notions of good and many elementary submodels and nice embeddings lead to different principles. The nice embeddings in the case of SEP and IDP are sep-embeddings and $\sigma$-embeddings, respectively.

Definition 1.1. Let $A$ and $B$ be Boolean algebras with $A \leq B$, i.e., $A$ is a subalgebra of $B$. For $b \in B$ let $A \uparrow b=\{a \in A: a \leq b\}$. Then $A \leq \leq_{\text {sep }} B$ if and only if for every $b \in B$ and every uncountable set $T \subseteq A\lceil b$ there is $a \in A\left\lceil b\right.$ such that $\{c \in T: c \leq a\}$ is uncountable. Let $A \leq_{\sigma} B$ if and only if for every $b \in B, A\lceil b$ has a countable cofinal subset.

Observe that $A \leq_{\sigma} B$ implies $A \leq_{\text {sep }} B$.

The good elementary submodels will be the same for both SEP and IDP. Later we show that $\leq_{\text {sep }}$ can be replaced by $\leq_{\sigma}$ in the following definition of SEP. The real difference between SEP and IDP lies in the interpretation of many.

Extending the notions defined in [2] and [9], we regard SEP and IDP as properties of general Boolean algebras, not only of $\mathcal{P}(\omega)$.

Definition 1.2. For a cardinal $\chi$ let $\mathcal{M}_{\chi}$ be the set of elementary submodels $M$ of $\mathcal{H}_{\chi}$ such that $|M|=\aleph_{1}$ and $[M]^{\aleph_{0}} \cap M$ is cofinal in $[M]^{\aleph_{0}}$. For a Boolean algebra $A, \operatorname{SEP}(A)$ is the statement "for all sufficiently large regular $\chi$ there are cofinally many $M \in \mathcal{M}_{\chi}$ such that $A \cap M \leq_{\text {sep }} A$ ". The axiom SEP introduced in [9] is $\operatorname{SEP}(\mathcal{P}(\omega))$.

$A$ has the $\left(\aleph_{1}, \aleph_{0}\right)$-ideal property (IDP) if and only if $A \cap M \leq_{\sigma} A$ for all sufficiently large regular $\chi$ and all $M \in \mathcal{M}_{\chi}$ with $A \in M$. We write $\operatorname{IDP}(A)$ if $A$ has the IDP.

As usual, we identify an algebraic structure $\left(A, f_{1}, \ldots, f_{n}, R_{1}, \ldots, R_{m}\right)$ with its underlying set $A$. It should be made clear that by " $A \in M$ " we really mean $\left(A, f_{1}, \ldots, f_{n}, R_{1}, \ldots, R_{m}\right) \in M$. The role of the cardinal $\chi$ is analyzed in Section 8, where we calculate precisely when a regular cardinal is "sufficiently large" in the definition of SEP.

Since $\leq_{\sigma}$ is stronger than $\leq_{\text {sep }}$, for a Boolean algebra $A, \operatorname{IDP}(A)$ implies $\operatorname{SEP}(A)$. In Section 2 we show that the relation $\leq_{\text {sep }}$ in the definition of SEP can be replaced by $\leq_{\sigma}$, i.e., SEP and IDP are very similar. In Section 8 we observe that it does not make a difference in the definition of SEP if we replace "there are cofinally many $M \in \mathcal{M}_{\chi}$ " by "there is $M \in \mathcal{M}_{\chi}$ " or by "there are stationarily many (in $\left.\left[\mathcal{H}_{\chi}\right]^{\aleph_{1}}\right) M \in \mathcal{M}_{\chi}$ ". This shows the importance of the results of Sections 6 and 7, namely that SEP is really weaker than IDP. 
If the universe is not very complex, that is, if some very weak version of the $\square$-principle together with $\mathrm{cf}\left([\mu]^{\aleph_{0}}\right)=\mu^{+}$holds for all singular cardinals $\mu$ of countable cofinality, or if the Boolean algebras under consideration are small, then the $\left(\aleph_{1}, \aleph_{0}\right)$-ideal property is equivalent to the weak FreeseNation property studied in [7]:

Definition 1.3. A Boolean algebra $A$ has the weak Freese-Nation property (WFN) if and only if there is a function $f: A \rightarrow[A] \leq \aleph_{0}$ such that for all $a, b \in A$ with $a \leq b$ there is $c \in f(a) \cap f(b)$ with $a \leq c \leq b$. In this case $f$ is called a WFN-function for $A$. We write $\operatorname{WFN}(A)$ for " $A$ has the WFN".

It is easy to check that if $f$ is a WFN-function for $A$ and $B \leq A$ is closed under $f$, then $B \leq_{\sigma} A$. In [7] Fuchino, Koppelberg, and Shelah characterized the WFN using elementary submodels and $\sigma$-embeddings. They showed

TheOREM 1.4. A Boolean algebra $A$ has the WFN if and only if $A \cap M \leq_{\sigma}$ A for all sufficiently large $\chi$ and all $M \preccurlyeq \mathcal{H}_{\chi}$ with $\aleph_{1} \subseteq M$ and $A \in M$.

Since $\aleph_{1} \subseteq M$ for all $M \in \mathcal{M}_{\chi}$, it is clear that for a Boolean algebra $A$, $\operatorname{IDP}(A)$ follows from $\mathrm{WFN}(A)$. As mentioned above, IDP and WFN are equivalent in many cases. Some of these cases are captured by the following lemma, which follows from the results in [8].

Lemma 1.5. If $A$ is a Boolean algebra of size $<\aleph_{\omega}$ or if $0^{\#}$ does not exist, then $\operatorname{IDP}(A)$ holds if and only if $\operatorname{WFN}(A)$ does.

The formulation using $0^{\sharp}$ is chosen here just for simplicity. As mentioned above, what is really needed is only a certain very weak assumption at singular cardinals of countable cofinality. On the other hand, it is known that the lemma does not hold without any such additional assumption (see [8] or [5]).

In [6] many interesting consequences of $\operatorname{WFN}(\mathcal{P}(\omega))$ have been found. Concerning the combinatorics of the reals, a universe satisfying $\operatorname{WFN}(\mathcal{P}(\omega))$ behaves very similarly to a Cohen model. In particular, the values of the popular cardinal invariants of the continuum, that is, those studied in [1], have the same values in a model with $\operatorname{WFN}(\mathcal{P}(\omega))$ as in a Cohen model with the same size of the continuum.

Our characterization of SEP in terms of $\leq_{\sigma}$ rather than $\leq_{\text {sep }}$ shows that the axiom $\operatorname{SEP}(\mathcal{P}(\omega))$ is sufficient to determine at least some of the smaller cardinal invariants of the continuum.

Juhász and Kunen have already proved that another consequence of $\operatorname{WFN}(\mathcal{P}(\omega))$, the principle $C_{2}^{s}\left(\omega_{2}\right)$ introduced in [10], follows from the weaker assumption $\operatorname{SEP}(\mathcal{P}(\omega))$. 
2. SEP is similar to IDP. As mentioned above, $\leq_{\sigma}$ implies $\leq_{\text {sep }}$. If the subalgebras under consideration are of size $\leq \aleph_{1}$, then the two relations are in fact the same.

Lemma 2.1. Let $A$ and $B$ be Boolean algebras with $A \leq_{\text {sep }} B$ and $|A|=\aleph_{1}$. Then $A \leq_{\sigma} B$.

Proof. Let $b \in B$ and assume for a contradiction that $A\lceil b$ is not countably generated. Let $\left(a_{\alpha}\right)_{\alpha<\omega_{1}}$ enumerate $A\left\lceil b\right.$. By recursion on $\alpha<\omega_{1}$ define a sequence $\left(c_{\alpha}\right)_{\alpha<\omega_{1}}$ in $A\left\lceil b\right.$ such that for all $\alpha<\omega_{1}, a_{\alpha} \leq c_{\alpha}$ and $c_{\alpha}$ is not in the ideal of $A$ generated by $\left\{c_{\beta}: \beta<\alpha\right\}$.

Now let $T=\left\{c_{\alpha}: \alpha<\omega_{1}\right\}$. We claim that $T$ is a counterexample to $A \leq_{\text {sep }} B$. For let $a \in A\left\lceil b\right.$. Then there is $\beta<\omega_{1}$ with $a=a_{\beta}$. Thus $a_{\beta} \leq c_{\beta}$. By the construction of the sequence $\left(c_{\alpha}\right)_{\alpha<\omega_{1}}$, there are only countably many elements of $T$ below $c_{\beta}$. Hence, there are only countably many elements of $T$ below $a$, contradicting $A \leq_{\text {sep }} B$.

Thus, we have

Corollary 2.2. For every Boolean algebra $A, \operatorname{SEP}(A)$ holds if and only if for all sufficiently large regular $\chi$ there are cofinally many $M \in \mathcal{M}_{\chi}$ with $A \cap M \leq_{\sigma} A$.

In the rest of this article we will freely use Corollary 2.2 without referring to it explicitly.

Using the characterization in Corollary 2.2 , it is easily seen that many interesting consequences of $\operatorname{WFN}(\mathcal{P}(\omega))$ already follow from $\operatorname{SEP}(\mathcal{P}(\omega))$. In the proofs of most of the results in [6] it is only used that under $\operatorname{WFN}(\mathcal{P}(\omega))$, for some sufficiently large $\chi$ there are cofinally many $M \in \mathcal{M}_{\chi}$ with $\mathcal{P}(\omega) \cap M \leq{ }_{\sigma} \mathcal{P}(\omega)$. The following theorem collects some of the consequences of $\operatorname{SEP}(\mathcal{P}(\omega))$ that follow from the arguments given in [6].

A subset of $[\omega]^{\aleph_{0}}$ is called groupwise dense if it is closed under taking almost subsets and non-meager with respect to the topology on $[\omega]^{\aleph_{0}}$ inherited from $2^{\omega}$ when identifying $[\omega]^{\aleph_{0}}$ with a subset of $2^{\omega}$.

Theorem 2.3. Assume $\operatorname{SEP}(\mathcal{P}(\omega))$. Then the following cardinal invariants of the continuum are $\aleph_{1}$ :

(i) $\operatorname{non}(\mathcal{M})$, the smallest size of a non-meager subset of $\mathbb{R}$, and

(ii) $\mathfrak{g}$, the smallest size of a family of groupwise dense subsets of $[\omega]^{\aleph_{0}}$ with empty intersection.

Moreover, if $\mathrm{CH}$ fails, then $\operatorname{cov}(\mathcal{M})$, the minimal size of a family of meager subsets of $\mathbb{R}$ covering $\mathbb{R}$, is at least $\aleph_{2}$.

It was also proved in [6] that $\operatorname{WFN}(\mathcal{P}(\omega))$ implies that $\mathfrak{a}$, the smallest size of a maximal almost disjoint family in $\mathcal{P}(\omega)$, is $\aleph_{1}$. In the proof it is 
sufficient to assume $\operatorname{IDP}(\mathcal{P}(\omega))$. The situation with $\mathfrak{a}$ under $\operatorname{SEP}(\mathcal{P}(\omega))$ is more subtle and will be discussed in Section 8.

For more information about these cardinal invariants see e.g. [1].

3. $\operatorname{SEP}(\mathcal{P}(\omega))$ holds in Cohen models. In [5] it was shown that $\operatorname{WFN}(\mathcal{P}(\omega))$ can fail in a Cohen model, assuming the consistency of some very large cardinal. In [8] it was shown that large cardinal assumptions are necessary for this.

However, $\operatorname{SEP}(\mathcal{P}(\omega))$ is always true in a Cohen model. We include a proof of this fact (Theorem 3.1). It follows that $\operatorname{WFN}(\mathcal{P}(\omega)$ ) does not follow from $\operatorname{SEP}(\mathcal{P}(\omega))$ in ZFC (assuming the consistency of certain large cardinals). We do not know whether $\operatorname{IDP}(\mathcal{P}(\omega))$ is always true in a Cohen model.

In Section 7 we shall show, without large cardinal assumptions, that $\operatorname{SEP}(\mathcal{P}(\omega))$ does not imply $\operatorname{IDP}(\mathcal{P}(\omega))$.

TheOREM 3.1. Let $V$ be a model of $\mathrm{CH}$ and suppose that $G$ is $\operatorname{Fn}(\kappa, 2)$ generic over $V$. Then $V[G] \models \operatorname{SEP}(\mathcal{P}(\omega))$.

The proof of this theorem relies on the following series of lemmas. The first lemma was proved in [14].

Lemma 3.2. Let $M$ be a transitive model of set theory and let $x \subseteq \omega$ be a Cohen real over $M$. Then $\mathcal{P}(\omega) \cap M \leq_{\sigma} \mathcal{P}(\omega) \cap M[x]$.

Lemma 3.3. Let $P$ be a c.c.c. partial order and let $M \in \mathcal{M}_{\chi}$ be such that $P \in M$. Let $G$ be P-generic over the ground model $V$. Then $M[G] \in \mathcal{M}_{\chi}^{V[G]}$.

Proof. Let $M$ and $G$ be as above. Then $M[G] \preccurlyeq \mathcal{H}_{\chi}[G]$ by c.c.c. of $P$. Let $X \subseteq M[G]$ be countable. In $V[G]$, there is a countable set $C \subseteq M$ of $P$-names such that $X \subseteq\left\{\dot{x}_{G}: \dot{x} \in C\right\}$. Again by the c.c.c. of $P$, we may assume that $C \in V$ and $C$ is countable in $V$. By $M \in \mathcal{M}_{\chi}^{V}$, we may assume $C \in M$. Since $M$ contains a name for $G, G \in M[G]$ and thus $X \subseteq\left\{\dot{x}_{G}: \dot{x} \in C\right\} \in M[G]$.

Lemma 3.4. Assume CH. Then $M \in \mathcal{M}_{\chi}$ implies that $[M] \leq \aleph_{0} \subseteq M$. In particular, for all $M \in \mathcal{M}_{\chi}$, if $P \subseteq M$ is a c.c.c. partial order, then every nice $P$-name for a subset of $\omega$ is contained in $M$.

Proof. Suppose that $x \in[M]^{\aleph_{0}}$. By $M \in \mathcal{M}_{\chi}$ there is $y \in[M]^{\aleph_{0}} \cap M$ such that $x \subseteq y$. By CH there is a surjection $f: \omega_{1} \rightarrow \mathcal{P}(y)$. By elementarity, there is such an $f$ in $M$. Let $\alpha<\omega_{1}$ be such that $f(\alpha)=x$. Since $\omega_{1} \subseteq M$, we have $\alpha \in M$ and thus $x=f(\alpha) \in M$.

Now let $P \subseteq M$ be a c.c.c. partial order. Since $P$ is c.c.c., every nice $P$-name for a subset of $\omega$ is a countable subset of $\{\check{n}: n \in \omega\} \times P \subseteq M$. By the first part of the lemma, every such set is an element of $M$. 
Lemma 3.5. Let $V$ and $G$ be as in Theorem 3.1. Let $\chi \in V$ be large enough and let $M \in\left(\mathcal{M}_{\chi}\right)^{V}$ be such that $\kappa \in M$. Then

$$
\mathcal{P}(\omega) \cap M[G]=\mathcal{P}(\omega) \cap V[G \cap \operatorname{Fn}(\kappa \cap M, 2)] .
$$

Proof. First let $x \in \mathcal{P}(\omega) \cap M[G]$. In $M$ there is a name $\dot{x}$ for $x$ which is a nice name for a subset of $\omega$. Since $\operatorname{Fn}(\kappa, 2)$ satisfies the c.c.c., in $V$ there is a countable set $X \subseteq \kappa$ such that $\dot{x}$ is an $\operatorname{Fn}(X, 2)$-name. We can find such an $X$ in $M$. Since $\aleph_{0} \subseteq M, X \subseteq M$. Therefore $\dot{x}$ is an $\operatorname{Fn}(\kappa \cap M, 2)$-name and thus $x \in V[G \cap \operatorname{Fn}(\kappa \cap M, 2)]$.

For the converse let $x \in V[G \cap \operatorname{Fn}(\kappa \cap M, 2)]$. Pick a nice $\operatorname{Fn}(\kappa \cap M, 2)$ name $\dot{x}$ for $x$. Clearly, $\operatorname{Fn}(\kappa \cap M, 2) \subseteq M$. Therefore Lemma 3.4 applies, and we get $\dot{x} \in M$. This shows $x \in M[G]$.

Proof of Theorem 3.1. We argue in $V[G]$. Let $\chi$ be sufficiently large and let $M \in \mathcal{M}_{\chi}$. It is sufficient to show that there is $M^{\prime} \in \mathcal{M}_{\chi}$ with $M \subseteq M^{\prime}$ and $\mathcal{P}(\omega) \cap M^{\prime} \leq_{\sigma} \mathcal{P}(\omega)$.

Since $\operatorname{Fn}(\kappa, 2)$ has the c.c.c., in $V$ there is a set $X$ of $\operatorname{size} \aleph_{1}$ of $\operatorname{Fn}(\kappa, 2)$ names such that every element of $M$ has a name in $X$. Let $N \in \mathcal{M}_{\chi}^{V}$ be such that $X \subseteq N$. Clearly, $M \subseteq N[G]$. By Lemma 3.3, $N[G] \in \mathcal{M}_{\chi}$. By Lemma 3.5, $\mathcal{P}(\omega) \cap N[G]=\mathcal{P}(\omega) \cap V[G \cap \operatorname{Fn}(\kappa \cap N, 2)]$. Since $V[G]$ is an $\operatorname{Fn}(\kappa \backslash N, 2)$-generic extension over $V[G \nmid \operatorname{Fn}(\kappa \cap N, 2)]$, it follows from Lemma 3.2 that $\mathcal{P}(\omega) \cap N[G] \leq_{\sigma} \mathcal{P}(\omega)$. This shows that, in $V[G]$, the set of $M^{\prime} \in \mathcal{M}_{\chi}$ with $\mathcal{P}(\omega) \cap M^{\prime} \leq_{\sigma} \mathcal{P}(\omega)$ is cofinal in $\left[\mathcal{H}_{\chi}\right]^{\aleph_{1}}$.

4. IDP for partial orders. In [7] the WFN has been defined for partial orders, not only for Boolean algebras. In this section and the next, we do the same for SEP and IDP. We have to liberalize our definition of $\leq_{\sigma}$.

Definition 4.1. Let $P$ and $Q$ be partial orders with $Q \leq P$, i.e., $Q \subseteq P$ and the orders on $Q$ and $P$ agree on $Q$. For $p \in P$ let $Q\lceil p=\{q \in Q: q \leq p\}$ and $Q \uparrow p=\{q \in Q: q \geq p\}$. Now $Q \leq_{\sigma} P$ if and only if for all $p \in P, Q\lceil p$ has a countable cofinal subset and $Q \uparrow p$ has a countable coinitial subset.

It is clear that for Boolean algebras $A$ and $B$, if $A$ is a subalgebra of $B$, then $A \leq_{\sigma} B$ holds in the Boolean-algebraic sense if and only if $A \leq_{\sigma} B$ holds for the partial orders. Now we can extend the notions IDP, SEP, and WFN to partial orders.

Definition 4.2. For a partial order $P$ :

- $\operatorname{SEP}(P)$ holds if and only if for all sufficiently large regular $\chi$ there are cofinally many $M \in \mathcal{M}_{\chi}$ with $P \cap M \leq_{\sigma} P$.

- IDP $(P)$ holds if and only if $P \cap M \leq_{\sigma} P$ for all sufficiently large regular $\chi$ and for all $M \in \mathcal{M}_{\chi}$ with $P \in M$. 
- WFN $(P)$ holds if and only if $P \cap M \leq_{\sigma} P$ for all sufficiently large regular $\chi$ and all $M \preccurlyeq \mathcal{H}_{\chi}$ with $\aleph_{1} \subseteq M$ and $P \in M$.

It is easy to check that the extended versions of SEP, IDP, and WFN agree with the old ones on Boolean algebras. For SEP this relies on Corollary 2.2, for WFN on Theorem 1.4.

Let us look at the partial orders $\left.([\kappa]]^{\leq \aleph_{0}}, \subseteq\right)$. Assuming the consistency of some large cardinal, it was shown in $[8]$ that $\operatorname{WFN}\left(\left[\aleph_{\omega}\right]^{\leq \aleph_{0}}\right)$ does not follow from GCH. However, we have

ThEOREM 4.3. CH implies $\operatorname{IDP}\left([\kappa]^{\leq \aleph_{0}}\right)$ for every cardinal $\kappa$.

Proof. Assume $\mathrm{CH}$ and let $\chi$ be sufficiently large and regular. Let $M \in \mathcal{M}_{\chi}$ be such that $[\kappa]^{\leq \aleph_{0}} \in M$. Then we have $\kappa \in M$. By Lemma 3.4, $M \cap[\kappa] \leq \aleph_{0}=[\kappa \cap M] \leq \aleph_{0}$.

Thus it remains to show that $[\kappa \cap M]]^{\leq \aleph_{0}} \leq_{\sigma}[\kappa] \leq \aleph_{0}$. Suppose $x \in[\kappa] \leq \aleph_{0}$.

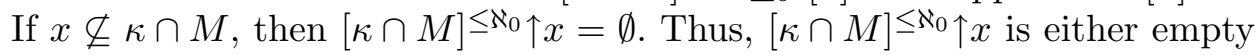
or has a minimal element, namely $x$.

$[\kappa \cap M] \stackrel{\aleph}{0}\lceil x$ always has a maximal element, namely $x \cap M$. This finishes the proof of the theorem.

This theorem can be regarded as a parallel of Theorem 3.1. It follows that, assuming the consistency of some large cardinal, it is consistent that there is a partial order that has the IDP, but not the WFN.

5. Complete Boolean algebras satisfying SEP. Just as the WFN, SEP is hereditary with respect to order retracts. A partial order $P$ is an order retract of a partial order $Q$ if there are order preserving maps $e: P \rightarrow Q$ and $f: Q \rightarrow P$ such that $f \circ e=\operatorname{id}_{P}$. If $P$ and $Q$ are Boolean algebras and $e$ and $f$ are (Boolean) homomorphisms, then we call $P$ a retract of $Q$.

Lemma 5.1. Let $P$ and $Q$ be partial orders such that $P$ is an order retract of $Q$. Then $\operatorname{SEP}(Q)$ implies $\operatorname{SEP}(P)$.

Proof. Let $\chi$ be large enough and $M \preccurlyeq \mathcal{H}_{\chi}$. Suppose $M \cap Q \leq_{\sigma} Q$ and $P, Q \in M$. We show $P \cap M \leq_{\sigma} P$.

Since $M$ knows that $P$ is a retract of $Q, M$ contains order preserving maps $e: P \rightarrow Q$ and $f: Q \rightarrow P$ such that $f \circ e=\operatorname{id}_{P}$. Let $p \in P$. Since $Q \cap M \leq{ }_{\sigma} Q$, there is a countable set $C \subseteq Q \cap M$ such that $C$ is cofinal in $Q \cap M\lceil e(p)$.

Claim. $f[C]$ is cofinal in $P \cap M\lceil p$.

Let $q \in P \cap M \uparrow p$. Since $e(q) \in Q \cap M \uparrow e(p)$, there is $c \in C$ such that $e(q) \leq c \leq e(p)$. Now $q \leq f(c) \leq p$, which proves the claim.

By the same argument, $P \cap M \uparrow p$ has a countable coinitial subset. This implies $\operatorname{SEP}(P)$. 
If $P$ is a complete lattice and $P$ embeds into $Q$ via $e$, then there is a map $f: Q \rightarrow P$ with $f \circ e=\operatorname{id}_{P}$, namely the map defined by $f(q)=$ $\sup \{p \in P: e(p) \leq q\}$ for all $q \in Q$. Thus, a complete lattice which embeds into a partial order $Q$ with $\operatorname{SEP}(Q)$ also has the property SEP.

Note that if $A$ is a complete Boolean algebra and $A$ embeds into $B$ in the Boolean-algebraic sense, then $A$ is a retract of $B$ by Sikorski's extension criterion. If $A$ is an infinite complete Boolean algebra, then $A$ has a maximal antichain of size $\aleph_{0}$ and $\mathcal{P}(\omega)$ embeds into $A$, again by Sikorski's extension criterion. Since $\mathcal{P}(\omega)$ is complete, it is a retract of $A$.

It follows that $\operatorname{SEP}(\mathcal{P}(\omega))$ holds if there is any infinite complete Boolean algebra $A$ with $\operatorname{SEP}(A)$. Note that for this it is not necessary to use Sikorski's criterion; our statement about complete lattices suffices.

While $\operatorname{SEP}(\mathcal{P}(\omega))$ is consistent with (but not a theorem of) ZFC, $\operatorname{SEP}\left(\mathcal{P}\left(\omega_{1}\right)\right)$ fails. We prove this in a series of lemmas. Together with Lemma 5.1 this will imply that all complete Boolean algebras $A$ with $\operatorname{SEP}(A)$ satisfy the c.c.c. It should be pointed out that the proof of $\neg \operatorname{WFN}\left(\mathcal{P}\left(\omega_{1}\right)\right)$ given in [7] also works for SEP. However, we believe that our argument is simpler.

In the following $\chi$ always denotes a sufficiently large regular cardinal.

LEMMA 5.2. (a) $\neg \operatorname{SEP}\left(\omega_{2}+1\right)$.

(b) If $\operatorname{SEP}(A)$ holds, then $A$ does not have a chain of order type $\omega_{2}$.

Proof. For (a) note that for each $M \in \mathcal{M}_{\chi}, \omega_{2} \cap M$ is an ordinal of cofinality $\aleph_{1}$. It follows that for each $\alpha \in \omega_{2} \backslash M,\left(\omega_{2}+1\right) \cap M\lceil\alpha$ has uncountable cofinality. In particular, $\left(\omega_{2}+1\right) \cap M \not_{\sigma} \omega_{2}+1$.

For (b) let $A$ be a Boolean algebra such that $\omega_{2}$ embeds into $A$. Clearly, $\omega_{2}+1$ also embeds into $A$. But $\omega_{2}+1$ is a complete lattice. Thus $\omega_{2}+1$ is an order retract of $A$. Now $\neg \operatorname{SEP}(A)$ follows from Lemma 5.1 together with part (a).

\section{LEMMA 5.3. $\neg \operatorname{SEP}\left(\mathcal{P}\left(\omega_{1}\right) /\left[\omega_{1}\right]^{\leq \aleph_{0}}\right)$.}

Proof. By Lemma 5.2, it suffices to show that $\mathcal{P}\left(\omega_{1}\right) /\left[\omega_{1}\right] \leq \aleph_{0}$ has a chain of order type $\omega_{2}$.

For $f, g \in \omega_{1}^{\omega_{1}}$ let $f<^{*} g$ if and only if $\left\{\alpha<\omega_{1}: f(\alpha) \geq g(\alpha)\right\}$ is countable. If $\left(f_{\gamma}\right)_{\gamma<\omega_{2}}$ is a $<^{*}$-increasing sequence in $\omega_{1}^{\omega_{1}}$, then $(\{(\alpha, \beta) \in$ $\left.\left.\omega_{1} \times \omega_{1}: \beta \leq f_{\gamma}(\alpha)\right\}\right)_{\gamma<\omega_{2}}$ gives rise to a strictly increasing sequence in $\mathcal{P}\left(\omega_{1} \times \omega_{1}\right) /\left[\omega_{1} \times \omega_{1}\right] \leq \aleph_{0}$ of order type $\omega_{2}$. It is thus sufficient to construct a $<^{*}$-increasing sequence of order type $\omega_{2}$ in $\omega_{1}^{\omega_{1}}$. But this is easy using the natural diagonalization argument to get an $<^{*}$-upper bound for any set $F \subseteq \omega_{1}^{\omega_{1}}$ of size $\leq \aleph_{1}$.

LEMMA 5.4. $\neg \operatorname{SEP}\left(\mathcal{P}\left(\omega_{1}\right)\right)$. 
Proof. Let $M \in \mathcal{M}_{\chi}$. Suppose $\mathcal{P}\left(\omega_{1}\right) \cap M \leq_{\sigma} \mathcal{P}\left(\omega_{1}\right)$. We show that

$$
\left(\mathcal{P}\left(\omega_{1}\right) /\left[\omega_{1}\right]^{\leq \aleph_{0}}\right) \cap M \leq_{\sigma} \mathcal{P}\left(\omega_{1}\right) /\left[\omega_{1}\right]^{\leq \aleph_{0}} .
$$

This suffices for the lemma since together with Lemma 5.3 it implies that there are not cofinally many $N \in \mathcal{M}_{\chi}$ with $\mathcal{P}\left(\omega_{1}\right) \cap N \leq_{\sigma} \mathcal{P}\left(\omega_{1}\right)$. Note that $\left(\mathcal{P}\left(\omega_{1}\right) \cap M\right) /\left(\left[\omega_{1}\right]^{\leq \aleph_{0}} \cap M\right)$ is essentially the same as $\left.\left(\mathcal{P}\left(\omega_{1}\right) /\left[\omega_{1}\right]\right]^{\leq \aleph_{0}}\right) \cap M$ since $M$ includes a cofinal subset of $\left[\omega_{1}\right]{ }^{\leq \aleph_{0}}$, namely the countable ordinals.

Let $a \in \mathcal{P}\left(\omega_{1}\right)$. Let $C$ be a cofinal subset of $\mathcal{P}\left(\omega_{1}\right) \cap M\lceil a$. Let $D$ be the set of all classes modulo $\left[\omega_{1}\right]^{\leq \aleph_{0}}$ of elements of $C$ and let $\bar{a}$ be the class of $a$ modulo $\left[\omega_{1}\right] \leq \aleph_{0}$.

Claim. $D$ is cofinal in $\left(\left(\mathcal{P}\left(\omega_{1}\right) /\left[\omega_{1}\right] \leq \aleph_{0}\right) \cap M\right)\lceil\bar{a}$.

Let $b \in \mathcal{P}\left(\omega_{1}\right) \cap M$ be such that $b \backslash a$ is countable. Let $\alpha<\omega_{1}$ be such that $b \backslash \alpha \subseteq a$. Since $\alpha \in M, b \backslash \alpha \in M$. Therefore, there is $c \in C$ such that $b \backslash \alpha \subseteq c \subseteq a$. The claim clearly follows from this. This finishes the proof of the lemma.

Let $A$ be a complete Boolean algebra not satisfying the c.c.c. Since $A$ is complete, $A$ has a maximal antichain of size $\aleph_{1}$. This antichain gives rise to an embedding of the algebra of finite-cofinite subsets of $\omega_{1}$ into $A$. Since $A$ is complete, this embedding extends to all of $\mathcal{P}\left(\omega_{1}\right)$ by Sikorski's extension criterion. Since $\mathcal{P}\left(\omega_{1}\right)$ is complete, it follows that $\mathcal{P}\left(\omega_{1}\right)$ is a retract of $A$. Using Lemma 5.1 this gives

Corollary 5.5. Let $A$ be a complete Boolean algebra. If $\operatorname{SEP}(A)$ holds, then $A$ satisfies the c.c.c.

6. An example in ZFC. In this section we show in ZFC that there is a Boolean algebra $A$ which satisfies $\operatorname{SEP}(A)$ but not $\operatorname{IDP}(A)$. Our construction shows some similarities with various constructions of thin-tall Boolean algebras as, for example, in [3].

Definition 6.1. Let $E_{\aleph_{1}}^{\aleph_{2}}=\left\{\alpha<\omega_{2}: \operatorname{cf}(\alpha)=\aleph_{1}\right\}$. For all $\alpha \in E_{\aleph_{1}}^{\aleph_{2}}$ fix an increasing sequence $\left(\delta_{\beta}^{\alpha}\right)_{\beta<\omega_{1}}$ which is cofinal in $\alpha$ and consists of successor ordinals. For $S \subseteq E_{\aleph_{1}}^{\aleph_{2}}$ let $A^{S}$ be the Boolean algebra defined as follows: Let $A_{0}^{S}=\{0,1\}$. Suppose $\alpha<\omega_{2}$ is a limit ordinal and $A_{\beta}^{S}$ has already been defined for all $\beta<\alpha$. Let $A_{\alpha}^{S}=\bigcup_{\beta<\alpha} A_{\beta}^{S}$. Now suppose $A_{\alpha}^{S}$ has been defined and $\alpha \in \omega_{2} \backslash S$. Let $A_{\alpha+1}^{S}=A_{\alpha}^{S}\left(x_{\alpha}\right)$ where $x_{\alpha}$ is independent over $A_{\alpha}^{S}$. Suppose that $A_{\alpha}^{S}$ has already been defined and $\alpha \in S$. Let $A_{\alpha+1}^{S}=A_{\alpha}^{S}\left(x_{\alpha}\right)$ where $x_{\alpha} \notin A_{\alpha}^{S}, A_{\alpha}^{S}\left\lceil x_{\alpha}\right.$ is generated by $\left\{x_{\delta_{\beta}^{\alpha}}: \beta<\omega_{1}\right\}$ and $A_{\alpha}^{S \uparrow-x_{\alpha}}$ is $\{0\}$. Finally let $A^{S}=\bigcup_{\alpha<\omega_{2}} A_{\alpha}^{S}$.

Fix a sufficiently large regular cardinal $\chi$. Recall that for every $M \in \mathcal{M}_{\chi}$, $\omega_{1} \subseteq M$, and thus $M \cap \omega_{2} \in \omega_{2}$. 
Lemma 6.2. Let $X \subseteq \mathcal{H}_{\chi}$ be of size $\aleph_{1}$. Then $C_{X}=\left\{M \cap \omega_{2}\right.$ : $\left.M \in \mathcal{M}_{\chi} \wedge X \subseteq M\right\}$ includes an $\aleph_{1}$-club of $\omega_{2}$, that is, an unbounded set which is closed under limits of subsets of cofinality $\aleph_{1}$.

Proof. By recursion, we define an increasing sequence $\left(M_{\alpha}\right)_{\alpha<\omega_{2}}$ in $\mathcal{M}_{\chi}$ such that

(i) $X \subseteq M_{0}$,

(ii) for all $\alpha<\omega_{2}, \alpha \in M_{\alpha}$, and

(iii) if $\alpha<\omega_{2}$ is a limit ordinal of cofinality $\aleph_{1}$, then $M_{\alpha}=\bigcup_{\beta<\alpha} M_{\beta}$.

This construction can be carried out since $\mathcal{M}_{\chi}$ is cofinal in $\left[\mathcal{H}_{\chi}\right]^{\aleph_{1}}$ and closed under unions of chains of length $\omega_{1}$. Let $C=\left\{\omega_{2} \cap M_{\alpha}: \alpha<\omega_{2}\right\}$. Then $C$ is unbounded in $\omega_{2}$ by (ii) and closed under limits of subsets of cofinality $\aleph_{1}$ by (iii). By (i), $C \subseteq C_{X}$.

From Lemma 6.2 we get

Lemma 6.3. Let $S$ be a stationary subset of $\omega_{2}$ such that $S \subseteq E_{\aleph_{1}}^{\aleph_{2}}$. Then for cofinally many $M \in \mathcal{M}_{\chi}$ we have $\omega_{2} \cap M \in S$.

Proof. Let $X \in\left[\mathcal{H}_{\chi}\right]^{\aleph_{1}}$. By Lemma 6.2 , the set $C_{X}$ includes an $\aleph_{1}$-club $C$ of $\omega_{2}$. Let $\bar{C}$ be the closure of $C$, i.e., $C$ together with all limit points of $C$. Then $\bar{C}$ is club in $\omega_{2}$ and $C=\bar{C} \cap E_{\aleph_{1}}^{\aleph_{2}}$. Since $S$ is stationary and a subset of $E_{\aleph_{1}}^{\aleph_{2}}, \bar{C} \cap S=C \cap S$ is non-empty, and thus there is $M \in \mathcal{M}_{\chi}$ such that $X \subseteq M$ and $M \cap \omega_{2} \in S$.

Using Lemma 6.3, we can show that for a suitably chosen set $S$, the Boolean algebra $A^{S}$ constructed above satisfies SEP but not IDP.

Theorem 6.4. There is a Boolean algebra $A$ with $\operatorname{SEP}(A)$ but not $\operatorname{IDP}(A)$.

Proof. Fix two disjoint stationary subsets $S_{0}$ and $S_{1}$ of $\omega_{2}$ with $S_{0} \cup S_{1}=$ $E_{\aleph_{1}}^{\aleph_{2}}$. Let $A=A^{S_{1}}$.

Claim 1. $\operatorname{SeP}(A)$.

Let $M \in \mathcal{M}_{\chi}$ be such that $\alpha=M \cap \omega_{2} \in S_{0}$ and $A \in M$. Then $A \cap M=A_{\alpha}^{S_{1}}$. Since $\alpha \notin S_{1}$ and by the construction of $A^{S_{1}}, A_{\alpha}^{S_{1}} \leq_{\sigma} A$. This proves the claim since there are cofinally many $M \in \mathcal{M}_{\chi}$ with $M \cap \omega_{2} \in S_{0}$ and $A \in M$ by Lemma 6.3 .

Claim 2. $\neg \operatorname{IDP}(A)$.

By Lemma 6.3, there is $M \in \mathcal{M}_{\chi}$ such that $\alpha=M \cap \omega_{2} \in S_{1}$ and $A \in M$. As above, $A \cap M=A_{\alpha}^{S_{1}}$. By the construction of $A^{S_{1}}, A_{\alpha}^{S_{1}} \not_{\sigma} A$. In other words, $M$ witnesses the failure of $\operatorname{IDP}(A)$. 
7. $\operatorname{SEP}(\mathcal{P}(\omega))$ does not imply $\operatorname{IDP}(\mathcal{P}(\omega))$. In this section we use the idea of the proof of Theorem 6.4 to construct a model of set theory where $\operatorname{SEP}(\mathcal{P}(\omega))$ holds while $\operatorname{IDP}(\mathcal{P}(\omega))$ fails. Our forcing construction is related to Shelah's oracle chain condition forcing [13] and to the innocuous forcing iterations introduced by Just [12]. In particular, the proof of Lemma 7.3 given below relies on the fact that certain tails of our forcing iteration are what is called harmless in [12].

ThEOREM 7.1. It is consistent that $\operatorname{SEP}(\mathcal{P}(\omega))$ holds but $\operatorname{IDP}(\mathcal{P}(\omega))$ fails.

Proof. Suppose the ground model $V$ satisfies $\mathrm{CH}$ and let $S_{0}$ and $S_{1}$ be as in the proof of Theorem 6.4. For each $\alpha \in E_{\aleph_{1}}^{\aleph_{2}}$ let $\left(\delta_{\beta}^{\alpha}\right)_{\beta<\omega_{1}}$ be as in Definition 6.1.

Our strategy is to perform a finite support iteration of c.c.c. forcings over $V$ of length $\omega_{2}$ where we add only Cohen reals most of the time. However, at stage $\alpha \in S_{1}$ we add a new subset $x_{\alpha}$ of $\omega$ such that the Cohen reals added at the stages $\delta_{\beta}^{\alpha}, \beta<\omega_{1}$, are almost contained in $x_{\alpha}$. Note that we consider the Cohen reals to be subsets of $\omega$. This construction should be viewed as the forcing version of the construction in the proof of Theorem 6.4.

We now define the iteration $\left(P_{\alpha}, Q_{\alpha}\right)_{\alpha<\omega_{2}}$. The underlying sets of the $Q_{\alpha}$ 's will be absolute, but not the orders. Thus we will not define each $Q_{\alpha}$ as a $P_{\alpha}$-name but as the underlying set of $Q_{\alpha}$ in $V$, also named $Q_{\alpha}$, together with a $P_{\alpha}$-name $\dot{\leq}_{\alpha}$ for the order on $Q_{\alpha}$.

For all $\alpha \notin S_{1}$ let $Q_{\alpha}$ be Cohen forcing, i.e., $\operatorname{Fn}(\omega, 2)$. Let $\dot{\leq}_{\alpha}$ be the canonical name for the usual order on $\operatorname{Fn}(\omega, 2)$, i.e., reverse inclusion. For

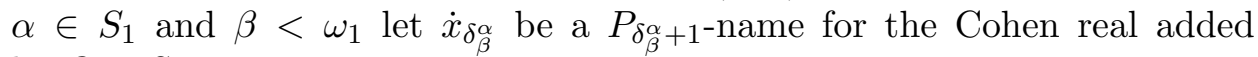
by $Q_{\delta_{\beta}^{\alpha}}$. Set

$$
Q_{\alpha}=\left\{(f, F): f \in \epsilon^{<\omega_{2}} 2, F \in\left[\left\{\delta_{\beta}^{\alpha}: \beta \in \omega_{1}\right\}\right]^{<\aleph_{0}}\right\}
$$

and let $\dot{\leq}_{\alpha}$ be a name for a relation $\leq$ on $Q_{\alpha}$ such that $(f, F) \leq\left(f^{\prime}, F^{\prime}\right)$ if and only if $f^{\prime} \subseteq f, F^{\prime} \subseteq F$, and for all $\delta \in F^{\prime}$, if $n \in \dot{x}_{\delta} \cap \operatorname{dom}\left(f \backslash f^{\prime}\right)$, then $f(n)=1$.

As usual, for each $\alpha<\omega_{2}$ let $P_{\alpha}$ be the finite support iteration of the $Q_{\beta}, \beta<\alpha$, where each $Q_{\alpha}=\left(Q_{\alpha}, \dot{\leq}_{\alpha}\right)$ is considered as a $P_{\alpha}$-name for the appropriate partial order. Let $P$ be the direct limit of the $P_{\alpha}, \alpha<\omega_{2}$. For convenience, by the absoluteness of the elements of the $Q_{\alpha}$ 's, we may assume that the elements of each $P_{\alpha}$ and of $P$ are elements of $\prod_{\beta<\omega_{2}} Q_{\beta}$ with finite support. For each condition $p \in P$ let $\operatorname{supt}(p)$ be its support.

Note that $P$ is c.c.c. since the $Q_{\alpha}$ 's are $\sigma$-centered. Let $G$ be $P$-generic over $V$. For $\alpha<\omega_{2}$ let $G_{\alpha}=G \cap P_{\alpha}$.

The easier part of the proof of the theorem is to show that in $V[G]$, $\operatorname{IDP}(\mathcal{P}(\omega))$ fails. To see this, we need 
Lemma 7.2. For $\alpha \in S_{1}, V[G] \models \mathcal{P}(\omega) \cap V\left[G_{\alpha}\right] \not E_{\sigma} \mathcal{P}(\omega)$.

Proof. We argue in $V[G]$. Let $\alpha \in S_{1}$. Note that $\mathcal{P}(\omega) \cap V\left[G_{\alpha}\right] \leq_{\sigma} \mathcal{P}(\omega)$ if and only if $\left(\mathcal{P}(\omega) \cap V\left[G_{\alpha}\right]\right) /$ fin $\leq_{\sigma} \mathcal{P}(\omega) /$ fin.

For $y \in \mathcal{P}(\omega)$ let $\bar{y}$ be the equivalence class of $y$ modulo fin. Let $x$ be the subset of $\omega$ generically added by $Q_{\alpha}$.

Claim. $\left(\mathcal{P}(\omega) \cap V\left[G_{\alpha}\right]\right) /$ fin $\lceil\bar{x}$ is generated by the classes modulo fin of the Cohen reals $\left\{x_{\delta_{\beta}^{\alpha}}: \beta<\omega_{1}\right\}$ added by the $Q_{\delta_{\beta}^{\alpha}}$ 's. In particular, $\left(\mathcal{P}(\omega) \cap V\left[G_{\alpha}\right]\right) /$ fin $\underline{L}_{\sigma} \mathcal{P}(\omega) /$ fin.

It follows from the construction of $Q_{\alpha}$ that for all $\beta<\omega_{1}, \bar{x}_{\delta_{\beta}^{\alpha}} \leq \bar{x}$. Let $a \in \mathcal{P}(\omega) \cap V\left[G_{\alpha}\right]$. Suppose that $a$ is not almost included in the union of a finite subset of $\left\{x_{\delta_{\alpha}^{\beta}}: \beta<\omega_{1}\right\}$. Then for every $n \in \omega$ the set of conditions in $Q_{\alpha}$ which force that there is $m \geq n$ such that $m \in a$ but $m \notin x$ is easily seen to be dense in $Q_{\alpha}$. It follows that $a$ is not almost included in $x$. This shows the claim and finishes the proof of Lemma 7.2.

By Lemma 6.3, in $V$ there is $M \in \mathcal{M}_{\chi}$ containing $P$ such that $\alpha=$ $M \cap \omega_{2} \in S_{1}$. Now $M[G] \cap \omega_{2}=\alpha$ since $P$ is c.c.c. If $\dot{x} \in M$ is a $P$-name for a subset of $\omega$, then $M$ also contains a nice $P$-name $\dot{y}$ for the same subset of $\omega$. By c.c.c., $\dot{y}$ only uses countably many conditions from $P$. These conditions are already contained in $P_{\beta}$ for some $\beta<\alpha$. Since $V$ satisfies $\mathrm{CH}$ and since $P_{\beta} \in M$ (and thus $\left.P_{\beta} \subseteq M\right), \dot{y} \in M$ by Lemma 3.4. It follows that $\mathcal{P}(\omega) \cap M[G]=\mathcal{P}(\omega) \cap V\left[G_{\alpha}\right]$. Therefore, $M[G]$ shows that $\mathcal{P}(\omega)$ does not satisfy IDP in $V[G]$.

To see that $\operatorname{SEP}(\mathcal{P}(\omega))$ holds in $V[G]$ we need

LEMMA 7.3. If $\alpha<\omega_{2}$ and $\alpha \notin S_{1}$, then $V[G]=\mathcal{P}(\omega) \cap V\left[G_{\alpha}\right] \leq_{\sigma} \mathcal{P}(\omega)$.

Proof. By Lemma 3.2, it is sufficient to show that for $\alpha \in \omega_{2} \backslash S_{1}$ every real in $V[G] \backslash V\left[G_{\alpha}\right]$ is contained in a Cohen extension of $V\left[G_{\alpha}\right]$.

Let $\alpha \in \omega_{2} \backslash S_{1}$. Let $x \in \mathcal{P}(\omega)$, but $x \notin V\left[G_{\alpha}\right]$. Then there is a $P$-name $\dot{x} \in V$ for $x$. By c.c.c., we may assume that $\dot{x}$ uses only countably many conditions from $P$. Our plan is to find $P_{\dot{x}} \subseteq P$ such that

(1) $P_{\alpha} \subseteq P_{\dot{x}}$ and $P_{\alpha}$ is completely embedded in $P_{\dot{x}}$,

(2) $\dot{x}$ is a $P_{\dot{x}}$-name,

(3) $P_{\dot{x}}$ is completely embedded in $P$, and

(4) the quotient $P_{\dot{x}}: G_{\alpha}$ is equivalent to $\operatorname{Fn}(\omega, 2)$.

This suffices for the lemma. For suppose $P_{\dot{x}}$ is as above. It is not hard to see that (3) implies that $P_{\dot{x}}: G_{\alpha}$ is completely embedded in $P: G_{\alpha}$. Note that by (1), it is reasonable to consider $P_{\dot{x}}: G_{\alpha}$. By $(2), \dot{x}$ can be regarded as a $P_{\dot{x}}: G_{\alpha}$-name. Thus by $(4), x$ is contained in a Cohen extension of $V\left[G_{\alpha}\right]$ and the lemma follows. 
It remains to construct $P_{\dot{x}}$ and to show that it has the required properties. For a condition $p \in P$ let

$$
\overline{\operatorname{supt}(p)}=\operatorname{supt}(p) \cup \bigcup\left\{F: \exists \beta \in \operatorname{supt}(p) \cap S_{1} \exists f(p(\beta)=(f, F))\right\} .
$$

Let

$$
X=\alpha \cup \bigcup\{\operatorname{supt}(p): \dot{x} \text { uses the condition } p\}
$$

and $P_{\dot{x}}=\{p \in P: \overline{\operatorname{supt}(p)} \subseteq X\}$.

Claim. $P_{\dot{x}}$ has properties (1)-(4).

(1) $P_{\alpha} \subseteq P_{\dot{x}}$ follows from the definitions. $P_{\dot{x}}$ can be viewed as finite support iteration which has $P_{\alpha}$ as an initial segment. Thus $P_{\alpha}$ is completely embedded in $P_{\dot{x}}$.

(2) It follows from the definitions that $\dot{x}$ is a $P_{\dot{x}}$-name.

(3) We have to show the following:

(i) $\forall p, q \in P_{\dot{x}}\left(p \perp_{P_{\dot{x}}} q \Rightarrow p \perp_{P} q\right)$,

(ii) $\forall p \in P \exists q \in P_{\dot{x}} \forall r \in P_{\dot{x}}\left(r \leq q \Rightarrow r \not \chi_{P} p\right)$.

For (i) observe that for all $p, q \in P$ with $p \not \perp_{P} q$ there is $r \in P$ such that $r \leq p, q$ and $\overline{\operatorname{supt}(r)} \subseteq \overline{\operatorname{supt}(p)} \cup \overline{\operatorname{supt}(q)}$. Therefore, if $p, q \in P_{\dot{x}}$ are compatible in $P$, then they are in $P_{\dot{x}}$.

(ii) is what really requires work. Let $p \in P$. Let $q^{\prime} \in P_{\dot{x}}$ be the condition with support $\operatorname{supt}(p) \cap X$ such that for all $\beta \in(\operatorname{supt}(p) \cap X) \backslash S_{1}, q^{\prime}(\beta)=p(\beta)$ and for all $\beta \in \operatorname{supt}(p) \cap X \cap S_{1}, q^{\prime}(\beta)=(f, F \cap X)$ where $f$ and $F$ are such that $p(\beta)=(f, F)$. This $q^{\prime}$ does not yet work for $q$ in (ii). We have to extend it a little.

Let $q$ be the condition with the same support as $q^{\prime}$ such that $q(\beta)=$ $q^{\prime}(\beta)$ for all $\beta \in \operatorname{supt}\left(q^{\prime}\right) \backslash S_{1}$. Now fix $\beta \in \operatorname{supt}\left(q^{\prime}\right) \cap S_{1}$. Let $f$ and $F$ be such that $q^{\prime}(\beta)=(f, F)$ Let $m \in \omega$ be such that for all $\gamma \in \operatorname{supt}(p) \backslash S_{1}$, $\operatorname{dom}(p(\gamma)) \subseteq m$. Let $g \in 2^{<\omega}$ be such that $m \subseteq \operatorname{dom}(g), f \subseteq g$, and $g(n)=1$ for all $n \in \operatorname{dom}(g) \backslash \operatorname{dom}(f)$. Now set $q(\beta)=(g, F)$.

SubClaim. $q$ works for (ii).

Let $r \in P_{\dot{x}}$ be such that $r \leq q$. We have to construct a common extension $s \in P$ of $p$ and $r$. As above, we build an approximation $s^{\prime}$ of $s$ first. For $\beta \in S_{1}$ with $p(\beta)=(f, F)$ and $r(\beta)=\left(f^{\prime}, F^{\prime}\right)$ let $s^{\prime}(\beta)=\left(f \cup f^{\prime}, F \cup F^{\prime}\right)$. Note that $f \cup f^{\prime}$ is a function since by the definition of $q$ and by $r \leq q$, we even have $f \subseteq f^{\prime}$ whenever $\beta \in \operatorname{supt}(r)$. Note that this definition makes sense if $\beta \notin \operatorname{supt}(r) \cap \operatorname{supt}(p)$ since the largest element of $Q_{\beta}$ is simply $(\emptyset, \emptyset)$ (for $\beta \in S_{1}$ ).

For $\beta \in \omega_{2} \backslash S_{1}$ let $s^{\prime}(\beta)=p(\beta) \cup r(\beta)$. Again, $p(\beta) \cup r(\beta)$ is a function since for $\beta \in \operatorname{supt}(r), p(\beta) \subseteq r(\beta)$ by $r \leq q$ and the definition of $q$. It is easy to see that $s^{\prime}$ extends $r$. 
It may happen that $s^{\prime} \not \leq p$. However, we can extend $s^{\prime}$ to a condition $s \leq p$ by adding some Cohen conditions (deciding more of the Cohen reals involved). Let $s(\beta)=s^{\prime}(\beta)$ for all $\beta \in S_{1}$. For $\beta \in S_{1}$ we have to make sure that $s\lceil\beta$ forces $s(\beta)$ to be below $p(\beta)$.

For all $\beta \in S_{1}$ let $f_{\beta}, F_{\beta}, f_{\beta}^{\prime}$, and $F_{\beta}^{\prime}$ be such that $s(\beta)=\left(f_{\beta}, F_{\beta}\right)$ and $p(\beta)=\left(f_{\beta}^{\prime}, F_{\beta}^{\prime}\right)$. Then for all $\beta \in S_{1}$ we have $f_{\beta}^{\prime} \subseteq f_{\beta}$ and $F_{\beta}^{\prime} \subseteq F_{\beta}$. For all $\delta \in F_{\beta}^{\prime}$ we want to have $\delta \in \operatorname{supt}(s)$ and $s(\delta) \Vdash \forall n \in \operatorname{dom}\left(f_{\beta}\right) \backslash \operatorname{dom}\left(f_{\beta}^{\prime}\right)$ $\left(f_{\beta}(n) \geq \dot{x}_{\delta}(n)\right)$. This can be accomplished. Just let $z$ be a sufficiently long finite sequence of zeros and put $s(\delta)=s^{\prime}(\delta) \frown z$ for every $\delta \in \bigcup\left\{F_{\beta}\right.$ : $\left.\beta \in \operatorname{supt}\left(s^{\prime}\right) \cap S_{1}\right\}$. Note that there are only finitely many $\delta$ 's to be considered. For every $\beta \in \omega_{2}$ for which $s(\beta)$ has not yet been defined let $s(\beta)=s^{\prime}(\beta)$.

It is straightforward to check that $s$ is a common extension of $r$ and $p$. This completes the proof of the subclaim and thus shows that $P_{\dot{x}}$ is completely embedded in $P$.

(4) Note that any two elements of $P_{\dot{x}}$ that agree on $\left[\alpha, \omega_{2}\right)$ are equivalent in $P_{\dot{x}}: G_{\alpha}$, i.e., they will be identified in the completion of $P_{\dot{x}}: G_{\alpha}$. But since $\left\{\delta_{\gamma}^{\beta}: \gamma \in \omega_{1}\right\} \cap \alpha$ is countable for all $\beta \in\left[\alpha, \omega_{2}\right) \cap S_{1}$ and $X \backslash \alpha$ is countable, there are only countably many possibilities for $p \uparrow\left[\alpha, \omega_{2}\right)$ for $p \in P_{\dot{x}}$. Therefore, the completion of $P_{\dot{x}}: G_{\alpha}$ has a countable dense subset. Since below each element of $P_{\dot{x}}: G_{\alpha}$ there are two incompatible elements (in $\left.P_{\dot{x}}: G_{\alpha}\right), P_{\dot{x}}: G_{\alpha}$ is equivalent to $\operatorname{Fn}(\omega, 2)$. This finishes the proof of the claim and of the lemma.

Since there are cofinally many $M \in\left(\mathcal{M}_{\chi}\right)^{V}$ with $M \cap \omega_{2} \in S_{0}$ by Lemma 6.3, the set $\left\{M[G]: M \in \mathcal{M}_{\chi} \wedge M \cap \omega_{2} \in S_{0}\right\}$ is cofinal in $\left(\mathcal{M}_{\chi}\right)^{V[G]}$. As above, for all $M \in\left(\mathcal{M}_{\chi}\right)^{V}, \alpha=M[G] \cap \omega_{2}=M \cap \omega_{2}$ and $\mathcal{P}(\omega) \cap M[G]=\mathcal{P}(\omega) \cap V\left[G_{\alpha}\right]$. Now it follows from Lemma 7.3 that $\operatorname{SEP}(\mathcal{P}(\omega))$ holds in $V[G]$. This finishes the proof of the theorem.

8. Variants of SEP. It is tempting to define a new class of partial orders by replacing "cofinally many $M \in \mathcal{H}_{\chi}$ " in the definition of SEP by "stationarily many $M \in \mathcal{H}_{\chi}$ ". However, the class of partial orders with this modified notion of SEP coincides with the class of partial orders with the original SEP. Also, one arrives at the same notion if "there are cofinally many $M \in \mathcal{H}_{\chi}$ " is weakened to "there is $M \in \mathcal{H}_{\chi}$ ".

For a partial order $P$ and a regular cardinal $\chi$ such that $P \in \mathcal{H}_{\chi}$ let

$$
\mathcal{M}(P, \chi)=\left\{M \in \mathcal{M}_{\chi}: P \in M \wedge P \cap M \leq_{\sigma} P\right\} .
$$

Theorem 8.1. Let $P$ be any partial order. Then the following are equivalent for $\kappa=\max \left(|\operatorname{trcl}(P)|, \aleph_{1}\right)$ : 
(1) $\operatorname{SEP}(P)$.

(2) There is a regular cardinal $\chi>\kappa$ such that $\mathcal{M}(P, \chi)$ is stationary in $\left[\mathcal{H}_{\chi}\right]^{\aleph_{1}}$.

(3) For every regular cardinal $\chi>\kappa, \mathcal{M}(P, \chi)$ is stationary in $\left[\mathcal{H}_{\chi}\right]^{\aleph_{1}}$.

(4) There is a regular cardinal $\chi>2^{\kappa}$ such that $\mathcal{M}(P, \chi)$ is non-empty.

(5) For every regular cardinal $\chi>2^{\kappa}, \mathcal{M}(P, \chi)$ is non-empty.

Clearly, (1) follows from (3) and implies (4). The remaining part of Theorem 8.1 is a special case of the following lemma, which has nothing to do with $\sigma$-embeddings. For a set $A$, a family $\mathcal{F} \subseteq \mathcal{P}(A)$, and a regular cardinal $\chi$ with $A, \mathcal{F} \in \mathcal{H}_{\chi}$ let

$$
\mathcal{M}(A, \mathcal{F}, \chi)=\left\{M \in \mathcal{M}_{\chi}: A, \mathcal{F} \in M \wedge A \cap M \in \mathcal{F}\right\} .
$$

For a partial order $P, \mathcal{M}(P, \chi)$ is simply $\mathcal{M}\left(P,\left\{Q \subseteq P: Q \leq_{\sigma} P\right\}, \chi\right)$.

Lemma 8.2. Let $A$ be a set and $\mathcal{F} \subseteq \mathcal{P}(A)$. Then the following are equivalent for $\kappa=\max \left(|\operatorname{trcl}(A)|, \aleph_{1}\right)$ :

(1) There is a regular cardinal $\chi \geq \kappa^{+}$such that $\mathcal{M}(A, \mathcal{F}, \chi)$ is stationary in $\left[\mathcal{H}_{\chi}\right]^{\aleph_{1}}$.

(2) For every regular cardinal $\chi \geq \kappa^{+}, \mathcal{M}(A, \mathcal{F}, \chi)$ is stationary in $\left[\mathcal{H}_{\chi}\right]^{\aleph_{1}}$.

(3) There is a regular cardinal $\chi>2^{\kappa}$ such that $\mathcal{M}(A, \mathcal{F}, \chi)$ is nonempty.

(4) For every regular cardinal $\chi>2^{\kappa}, \mathcal{M}(A, \mathcal{F}, \chi)$ is non-empty.

The reason for considering $\kappa^{+}$and $2^{\kappa}$ in the formulation of this lemma is that $\kappa^{+}$is the least cardinal $\chi>\aleph_{1}$ with $A \in \mathcal{H}_{\chi}$ and the size of $\mathcal{H}_{\kappa^{+}}$ is $2^{\kappa}$. The proof of Lemma 8.2 uses two arguments: one for stepping up in cardinality and one for stepping down. We start with decreasing cardinals. Fix $A, \mathcal{F}$, and $\kappa$ as in Lemma 8.2.

Lemma 8.3. Let $\chi, \mu>\kappa$ be regular cardinals with $2^{<\chi}<\mu$. If $\mathcal{M}(A, \mathcal{F}, \mu)$ is non-empty, then $\mathcal{M}(A, \mathcal{F}, \chi)$ is stationary in $\left[\mathcal{H}_{\chi}\right]^{\aleph_{1}}$.

Proof. Suppose that $\mathcal{M}(A, \mathcal{F}, \chi)$ is not stationary in $\left[\mathcal{H}_{\chi}\right]^{\aleph_{1}}$. We may assume that $\chi>\kappa$ is minimal with this property. Let $M \in \mathcal{M}(A, \mathcal{F}, \mu)$.

Since $\left|\mathcal{H}_{\chi}\right|^{\aleph_{1}}=2^{<\chi}<\mu$, we have $\left[\mathcal{H}_{\chi}\right]^{\aleph_{1}}, \mathcal{M}(A, \mathcal{F}, \chi) \in \mathcal{H}_{\mu}$ and $\chi$ is definable in $\mathcal{H}_{\mu}$ with the parameters $A$ and $\mathcal{F}$. Therefore, $\chi \in M$ and $M$ knows that $\mathcal{M}(A, \mathcal{F}, \chi)$ is not stationary in $\left[\mathcal{H}_{\chi}\right]^{\aleph_{1}}$.

It follows that $M$ contains a club $\mathcal{C}$ of $\left[\mathcal{H}_{\chi}\right]^{\aleph_{1}}$ which is disjoint from $\mathcal{M}(A, \mathcal{F}, \chi)$. By elementarity, $M \cap \mathcal{H}_{\chi} \subseteq \bigcup(\mathcal{C} \cap M)$. Since $\aleph_{1} \subseteq M$, $\bigcup(\mathcal{C} \cap M) \subseteq M \cap \mathcal{H}_{\chi}$. Since $M \cap[M]^{\aleph_{0}}$ is cofinal in $[M]^{\aleph_{0}}, \mathcal{C} \cap M$ is countably directed. It follows that $\bigcup(M \cap \mathcal{C})$ is the union of an increasing chain of length $\omega_{1}$ of elements of $\mathcal{C}$. Therefore, $M \cap \mathcal{H}_{\chi}=\bigcup(\mathcal{C} \cap M) \in \mathcal{C}$. It is easily checked that $M \cap \mathcal{H}_{\chi} \in \mathcal{M}_{\chi}$. 
Since $A \subseteq \mathcal{H}_{\chi}, A \cap M \cap \mathcal{H}_{\chi}=A \cap M \in \mathcal{F}$. Thus, $M \cap \mathcal{H}_{\chi} \in \mathcal{C} \cap \mathcal{M}(A, \mathcal{F}, \chi)$, contradicting the choice of $\mathcal{C}$.

LEMmA 8.4. For all regular cardinals $\chi, \mu>\kappa$ with $\chi<\mu$, if $\mathcal{M}(A, \mathcal{F}, \chi)$ is stationary in $\left[\mathcal{H}_{\chi}\right]^{\aleph_{1}}$, then $\mathcal{M}(A, \mathcal{F}, \mu)$ is non-empty.

Proof. We use a refined Skolem hull operator to find $\widetilde{M} \in \mathcal{M}_{\mu}$ with $A, \mathcal{F} \in \widetilde{M}$ and $\widetilde{M} \cap A \in \mathcal{F}$. Fix a well-ordering $\sqsubset$ on $\mathcal{H}_{\mu}$. For $\alpha<\omega_{1}$ let $\mathrm{sk}_{\alpha}$ denote the Skolem hull operator on $\mathcal{H}_{\mu}$ with respect to the built-in Skolem functions of the structure $\left(\mathcal{H}_{\mu}, \in, \sqsubset, A, \mathcal{F}, \operatorname{sk}_{\beta}\right)_{\beta<\alpha}$ where $A$ and $\mathcal{F}$ are considered as constants. For $X \subseteq \mathcal{H}_{\chi}$ let

$$
\operatorname{sk}^{*}(X)=\bigcup\left\{\operatorname{sk}_{\alpha}(Y): Y \in X \wedge|Y| \leq \aleph_{0} \wedge \alpha<\omega_{1}\right\} .
$$

Claim. Let $X \in\left[\mathcal{H}_{\mu}\right]^{\aleph_{1}}$ be such that $X \cap[X] \leq \aleph_{0}$ is cofinal in $[X] \leq \aleph_{0}$. Then

(i) $X \subseteq \operatorname{sk}^{*}(X)$ and $A, \mathcal{F} \in \operatorname{sk}^{*}(X)$,

(ii) $\left|\operatorname{sk}^{*}(X)\right|=\aleph_{1}$,

(iii) $\left[\mathrm{sk}^{*}(X)\right]^{\leq \aleph_{0}} \cap \mathrm{sk}^{*}(X)$ is cofinal in $\left[\mathrm{sk}^{*}(X)\right]^{\leq \aleph_{0}}$, and

(iv) $\operatorname{sk}^{*}(X) \preccurlyeq \mathcal{H}_{\mu}$.

Moreover,

(v) for all $X, Y \subseteq \mathcal{H}_{\mu}$ with $X \subseteq Y$, $\mathrm{sk}^{*}(X) \subseteq \mathrm{sk}^{*}(Y)$ and

(vi) if $\left(X_{\alpha}\right)_{\alpha<\delta}$ is an increasing sequence of subsets of $\mathcal{H}_{\mu}$ and $X=$ $\bigcup_{\alpha<\delta} X_{\alpha}$, then $\operatorname{sk}^{*}(X)=\bigcup_{\alpha<\delta} \operatorname{sk}^{*}\left(X_{\alpha}\right)$.

For (i) let $x \in X$. By our assumptions on $X$, there is $Y \in X \cap[X] \leq \aleph_{0}$ such that $\{x\} \subseteq Y$. Now $x \in Y \subseteq \operatorname{sk}_{0}(Y)$ and thus $x \in \operatorname{sk}^{*}(X)$. This shows $X \subseteq \operatorname{sk}^{*}(X)$. Next, $A, \mathcal{F} \in \mathrm{sk}^{*}(X)$ since $A, \mathcal{F} \in \mathrm{sk}_{0}(Y)$ for every countable element $Y$ of $X$.

Statement (ii) follows from the fact that for every countable set $Y \subseteq \mathcal{H}_{\mu}$ and every $\alpha<\omega_{1}, \operatorname{sk}_{\alpha}(Y)$ is again countable.

For (iii) let $Y$ be a countable subset of $\operatorname{sk}^{*}(X)$. For every $n \in \omega$ fix $\alpha_{n}<\omega_{1}$ and a countable set $Y_{n} \in X$ such that $Y \subseteq \bigcup_{n \in \omega} \operatorname{sk}_{\alpha_{n}}\left(Y_{n}\right)$. By our assumptions on $X$, there is $Z \in X \cap[X]{ }^{\leq \aleph_{0}}$ such that $\left\{Y_{n}: n \in \omega\right\} \subseteq Z$. Let $\beta=\sup _{n \in \omega}\left(\alpha_{n}+1\right)$. Now for every $n \in \omega, \operatorname{sk}_{\alpha_{n}}\left(Y_{n}\right) \in \operatorname{sk}_{\beta}(Z)$ by the choice of $\operatorname{sk}_{\beta}$. Since $\operatorname{sk}_{\beta}(Z)$ is an elementary submodel of $\mathcal{H}_{\mu}$ and since the $\operatorname{sk}_{\alpha_{n}}\left(Y_{n}\right)$ are countable, for every $n \in \omega$ we also have $\operatorname{sk}_{\alpha_{n}}\left(Y_{n}\right) \subseteq \operatorname{sk}_{\beta}(Z)$. It follows that $Y \subseteq \operatorname{sk}_{\beta}(Z)$. Clearly, $\operatorname{sk}_{\beta}(Z)$ is a countable subset of $\operatorname{sk}^{*}(X)$. We are done with the proof of (iii) if we can $\operatorname{show} \operatorname{sk}_{\beta}(Z) \in \operatorname{sk}^{*}(X)$. But this is easy. Just let $Z^{\prime} \in X \cap[X] \leq \aleph_{0}$ be such that $Z \in Z^{\prime}$. Now $\operatorname{sk}_{\beta}(Z) \in$ $\operatorname{sk}_{\beta+1}\left(Z^{\prime}\right) \subseteq \operatorname{sk}^{*}(X)$.

For (iv) it suffices to show that for all finite subsets $F$ of $\operatorname{sk}^{*}(X)$ there is an elementary submodel $M$ of $\mathcal{H}_{\mu}$ such that $F \subseteq M \subseteq \operatorname{sk}^{*}(X)$. Let $F$ be a 
finite subset of $\operatorname{sk}^{*}(X)$. As before, there are $\beta<\omega_{1}$ and $Z \in X \cap[X] \leq \aleph_{0}$ such that $F \subseteq \operatorname{sk}_{\beta}(Z)$. By the definition of $\operatorname{sk}^{*}, \operatorname{sk}_{\beta}(Z) \subseteq \mathrm{sk}^{*}(X)$. And $\operatorname{sk}_{\beta}(Z)$ is an elementary submodel of $\mathcal{H}_{\mu}$.

Statements (v) and (vi) follow immediately from the definition of $\mathrm{sk}^{*}$. This finishes the proof of the claim.

Now consider the set

$$
\mathcal{C}=\left\{M \in\left[\mathcal{H}_{\chi}\right]^{\aleph_{1}}: \mathrm{sk}^{*}(M) \cap \mathcal{H}_{\chi}=M\right\} .
$$

From the properties of $\mathrm{sk}^{*}$ it follows that $\mathcal{C}$ is club in $\left[\mathcal{H}_{\chi}\right]^{\aleph_{1}}$. Since $\mathcal{M}(X, \mathcal{F}, \chi)$ is stationary, there is $M \in \mathcal{C} \cap \mathcal{M}(X, \mathcal{F}, \chi)$. Let $\widetilde{M}=\operatorname{sk}^{*}(M)$. By the properties of sk* $\widetilde{M} \in \mathcal{M}_{\chi}$ and $A, \mathcal{F} \in \widetilde{M}$. Moreover, $\widetilde{M} \cap X=$ $M \cap X \in \mathcal{F}$. In other words, $\widetilde{M} \in \mathcal{M}(X, \mathcal{F}, \mu)$.

Proof of Lemma 8.2. We start from (3). Suppose there is a regular cardinal $\chi>2^{\kappa}$ such that $\mathcal{M}(X, \mathcal{F}, \chi)$ is non-empty. Then, by Lemma 8.3, $\mathcal{M}\left(X, \mathcal{F}, \kappa^{+}\right)$is stationary in $\left[\mathcal{H}_{\kappa^{+}}\right]^{\aleph_{1}}$. This implies (1).

Now suppose that (1) holds. Then there is a regular cardinal $\chi>\kappa$ such that $\mathcal{M}(X, \mathcal{F}, \chi)$ is stationary in $\left[\mathcal{H}_{\chi}\right]^{\aleph_{1}}$. By Lemma 8.4, there are arbitrarily large regular cardinals $\mu$ such that $\mathcal{M}(X, \mathcal{F}, \mu)$ is non-empty. By Lemma 8.3, this implies that $\mathcal{M}(X, \mathcal{F}, \mu)$ is stationary for every regular $\mu>\kappa$, i.e., (2) holds. The implications $(2) \Rightarrow(4)$ and $(4) \Rightarrow(3)$ are trivial.

At the moment, we do not know whether $\mathfrak{a}=\aleph_{1}$ follows from $\operatorname{SEP}(\mathcal{P}(\omega))$. However, we can show that a variant of $\operatorname{SEP}(\mathcal{P}(\omega))$ which is called $\operatorname{SEP}^{+-}(\mathcal{P}(\omega))$ here (see below) implies $\mathfrak{a}=\aleph_{1}$.

In the following let $\chi$ always denote a regular cardinal.

DEFINITION 8.5.

$\mathcal{M}_{\chi}^{\sqsubset}=\left\{M: M \preccurlyeq \mathcal{H}_{\chi},|M|=\aleph_{1}\right.$, and there is a well-ordering $\sqsubset$ on $M$ of order type $\omega_{1}$ such that for every $\left.a \in M, \sqsubset \cap\left(M_{\sqsubset a}\right)^{2} \in M\right\}$ where $M_{\sqsubset a}=\{x \in M: x \sqsubset a\}$.

Definition 8.6. Let $P$ be a partial order.

(1) $\operatorname{SEP}^{+}(P)$ if $\left\{M \in \mathcal{M} \sqsubset \bar{\chi}: P \cap M \leq_{\sigma} P\right\}$ is cofinal in $\left[\mathcal{H}_{\chi}\right]^{\aleph_{1}}$ for every sufficiently large $\chi$.

(2) $\operatorname{SEP}^{+-}(P)$ if $\left\{M \in \mathcal{M} \sqsubset: P \cap M \leq_{\sigma} P\right\}$ is non-empty for a sufficiently large $\chi$.

For $A, \mathcal{F}, \chi$ as in the definition of $\mathcal{M}(A, \mathcal{F}, \chi)$, let

$$
\mathcal{M}^{\sqsubset}(A, \mathcal{F}, \chi)=\left\{M \in \mathcal{M}_{\chi}^{\sqsubset}: A, \mathcal{F} \in M \wedge A \cap M \in \mathcal{F}\right\} .
$$

Then it is easy to see that Lemma 8.3 with $\mathcal{M}^{\sqsubset}(A, \mathcal{F}, \chi)$ in place of $\mathcal{M}(A, \mathcal{F}, \chi)$ also holds. As in the proof of Lemma 8.2 , we obtain the following equivalence: 
$\operatorname{SEP}^{+}(P)$

$\Leftrightarrow\left\{M \in \mathcal{M}_{\chi}^{\sqsubset}: P \cap M \leq_{\sigma} P\right\}$ is stationary for every sufficiently large $\chi$

$\Leftrightarrow\left\{M \in \mathcal{M}_{\chi}^{\sqsubset}: P \cap M \leq_{\sigma} P\right\}$ is non-empty for every sufficiently large $\chi$.

Lemma 8.7. $\mathcal{M}_{\chi} \subseteq \mathcal{M}_{\chi}$.

Proof. Suppose that $M \in \mathcal{M}_{\chi}$ and $\sqsubset$ is a well-ordering of $M$ as in the definition of $\mathcal{M} \sqsubset$. For $X \in[M]^{\aleph_{0}}$ let $x \in M$ be such that $X \subseteq M_{\sqsubset x}$. Then $\left|M_{\sqsubset x}\right| \leq \aleph_{0}$ and $M_{\sqsubset x} \in M$. This shows that $[M]^{\aleph_{0}} \cap M$ is cofinal in $[M]^{\aleph_{0}}$. Hence $M \in \mathcal{M}_{\chi}$.

Lemma 8.8. Suppose that $M \in \mathcal{M} \sqsubset$. Then $M$ is internally approachable in the sense of [4], i.e.,

(*) $\quad M$ is the union of a continuously increasing chain of countable elementary submodels $\left(M_{\alpha}\right)_{\alpha<\omega_{1}}$ of $M$ such that $\left(M_{\beta}\right)_{\beta \leq \alpha} \in M_{\alpha+1}$ for all $\alpha<\omega_{1}$.

Proof. Suppose that $M \in \mathcal{M} \sqsubset \bar{\chi}$ and $\sqsubset$ is a well-ordering of $M$ as in the definition of $\mathcal{M} \sqsubset$.

Let $x_{\alpha} \in M, \alpha<\omega_{1}$ be defined inductively such that

(0) $M_{\sqsubset x_{\alpha}}$ is an elementary submodel of $(M, \in, \sqsubset)$.

(1) If $\alpha$ is a limit ordinal then $x_{\alpha}$ is the limit of $x_{\beta}, \beta<\alpha$.

(2) If $\alpha$ is a successor, say $\alpha=\beta+1$, then $x_{\alpha}$ is minimal with respect to $\sqsubset$ such that $x_{\beta} \sqsubset x_{\alpha}, \sqsubset \cap\left(M_{\sqsubset x_{\beta}}\right)^{2} \in M_{\sqsubset x_{\alpha}}$ and $(0)$.

Note that, in (2), the construction is possible since $\sqsubset \cap\left(M_{\sqsubset x_{\beta}}\right)^{2} \in M$ by the definition of $M \in \mathcal{M}_{\chi}$. By $(0)$ and since $\left(x_{\beta}\right)_{\beta \leq \alpha}$ is definable in $M$ with the parameter $\sqsubset \cap\left(M_{\sqsubset x_{\alpha}}\right)^{2}$ we have $\left(x_{\beta}\right)_{\beta \leq \alpha} \in M_{\sqsubset x_{\alpha+1}}$. It follows that $\left(M_{\sqsubset x_{\beta}}\right)_{\beta \leq \alpha} \in M_{\sqsubset x_{\alpha+1}}$. Also $M=\bigcup_{\alpha<\omega_{1}} M_{\sqsubset x_{\alpha}}$ since $\sqsubset$ has order type $\omega_{1}$. Thus $\left(M_{\sqsubset x_{\alpha}}\right)_{\alpha<\omega_{1}}$ is a sequence as required in $(*)$.

The property $(*)$ in Lemma 8.8 almost characterizes elements of $\mathcal{M}_{\chi}^{\sqsubset}$ :

Lemma 8.9. Let $<^{*}$ be a well-ordering of $\mathcal{H}_{\chi}$ of order type $\left|\mathcal{H}_{\chi}\right|$. If $\left(M_{\alpha}\right)_{\alpha<\omega_{1}}$ is a continuously increasing sequence of countable elementary submodels of $\left(\mathcal{H}_{\chi}, \in,<^{*}\right)$ such that $\left(M_{\beta}\right)_{\beta \leq \alpha} \in M_{\alpha+1}$ for all $\alpha<\omega_{1}$, then $M=\bigcup_{\alpha<\omega_{1}} M_{\alpha}$ is an element of $\mathcal{M}_{\chi}$.

Proof. For each $x \in M$, let $\alpha_{x}=\min \left\{\alpha<\omega_{1}: x \in M_{\alpha+1}\right\}$. Let $\sqsubset$ be the linear ordering on $M$ defined by

$$
x \sqsubset y \Leftrightarrow \alpha_{x}<\alpha_{y} \vee\left(\alpha_{x}=\alpha_{y} \wedge x<^{*} y\right) .
$$

Clearly $\sqsubset$ is a well-ordering on $M$. Moreover, $\sqsubset$ has order type $\omega_{1}$ since every initial segment of $M$ with respect to $\sqsubset$ is countable and $M$ itself is uncountable. 
Let $x \in M$. We show that $\sqsubset \cap\left(M_{\sqsubset x}\right)^{2} \in M$. Let $\alpha^{*}=\alpha_{x}+2$. In $M_{\alpha^{*}}, \sqsubset \cap$ $\left(M_{\sqsubset x}\right)^{2}$ is definable from $<^{*} \cap\left(M_{\alpha_{x}+1}\right)^{2}$ and $\left(M_{\beta}\right)_{\beta \leq \alpha_{x}+1}$. By elementarity and since $<^{*} \cap\left(M_{\alpha_{x}+1}\right)^{2}$ and $\left(M_{\beta}\right)_{\beta \leq \alpha_{x}+1}$ are elements of $M_{\alpha^{*}}$, it follows that $\sqsubset \cap\left(M_{\sqsubset x}\right)^{2} \in M_{\alpha^{*}} \subseteq M$.

By Lemmas 8.8 and 8.9 , there are club many $M \preccurlyeq \mathcal{H}_{\chi}$ of size $\aleph_{1}$ such that $M \in \mathcal{M}_{\chi}$ if and only if $M$ is internally approachable - namely those $M$ with $M \preccurlyeq\left(\mathcal{H}_{\chi}, \in,<^{*}\right)$ for some fixed $<^{*}$ as above.

LEMma 8.10. $\mathcal{M} \sqsubset$ is stationary in $\left[\mathcal{H}_{\chi}\right]^{\aleph_{1}}$.

Proof. Suppose that $\mathcal{C} \subseteq\left[\mathcal{H}_{\chi}\right]^{\aleph_{1}}$ is closed unbounded. We show that $\mathcal{M} \sqsubset \bar{\chi} \cap \neq \emptyset$.

Let $<^{*}$ be a well-ordering of $\mathcal{H}_{\chi}$ of order type $\left|\mathcal{H}_{\chi}\right|$. Let $\left(M_{\alpha}\right)_{\alpha<\omega_{1}}$ be a continuously increasing chain of countable elementary submodels of $\left(\mathcal{H}_{\chi}, \mathcal{C}, \in,<^{*}\right)$ such that $\left(M_{\beta}\right)_{\beta \leq \alpha} \in M_{\alpha+1}$ for all $\alpha<\omega_{1}$. Let $M=\bigcup_{\alpha<\omega_{1}} M_{\alpha}$. Then $M \in \mathcal{M}_{\chi}$ by Lemma 8.9. Since $\omega_{1} \subseteq M$, we have $N \subseteq M$ for all $N \in \mathcal{C} \cap M$. By elementarity $\mathcal{C} \cap M$ is a directed system and $M=\bigcup(\mathcal{C} \cap M)$. Since $|M|=\aleph_{1}$, it follows that $M \in \mathcal{C}$.

Lemma 8.11. For a partial order P:

(1) $\operatorname{IDP}(P)$ implies $\mathrm{SEP}^{+}(P)$,

(2) $\mathrm{SEP}^{+}(P)$ implies $\mathrm{SEP}^{+-}(P)$,

(3) $\operatorname{SEP}^{+-}(P)$ implies $\operatorname{SEP}(P)$,

that is,

$$
\mathrm{WFN}(P) \Rightarrow \mathrm{IDP}(P) \Rightarrow \mathrm{SEP}^{+}(P) \Rightarrow \mathrm{SEP}^{+-}(P) \Rightarrow \mathrm{SEP}(P) .
$$

Proof. (1) follows from Lemma 8.7. (2) is clear from definitions. (3) follows from Theorem 8.1.

Theorem 8.12. Assume $\operatorname{SEP}^{+-}(\mathcal{P}(\omega))$. Then $\mathfrak{a}=\aleph_{1}$.

Proof. Let $\chi$ be sufficiently large and $M^{*} \in \mathcal{M}_{\chi}$ be such that $\mathcal{P}(\omega) \cap$ $M^{*} \leq{ }_{\sigma} \mathcal{P}(\omega)$. Since $\left|M^{*}\right|=\aleph_{1}$, it is enough to show that there is a MAD family $\subseteq M^{*}$.

Let $\sqsubset$ be a well-ordering of $M^{*}$ as in the definition of $\mathcal{M}_{\chi}$. By Lemma 8.8, there is an increasing sequence $\left(M_{\alpha}\right)_{\alpha<\omega_{1}}$ of countable elementary submodels of $M^{*}$ such that $\bigcup_{\alpha<\omega_{1}} M_{\alpha}=M$ and $\left(M_{\beta}\right)_{\beta \leq \alpha} \in M_{\alpha+1}$ for all $\alpha<\omega_{1}$.

Let $\left(a_{\alpha}\right)_{\alpha<\omega_{1}}$ be such that

(1) $\left\{a_{n}: n \in \omega\right\}$ is a partition of $\omega$ with $\left(a_{n}\right)_{n \in \omega} \in M_{0}$;

(2) For $\alpha \geq \omega, a_{\alpha} \in[\omega]^{\aleph_{0}} \cap M_{\alpha+1}$ is minimal (with respect to $\sqsubset$ ) with the following properties:

(i) $a_{\alpha}$ is almost disjoint from each $a_{\beta}, \beta<\alpha$.

(ii) $\forall x \in[\omega]^{\aleph_{0}} \cap M_{\alpha}\left(\forall u \in[\alpha]^{<\aleph_{0}}\left(\left|x \backslash \bigcup_{\beta \in u} a_{\beta}\right|=\aleph_{0}\right) \rightarrow\left|a_{\alpha} \cap x\right|=\aleph_{0}\right)$. 
Notice that in $\mathcal{H}_{\chi}$ the sequence $\left(a_{\beta}\right)_{\beta<\alpha}$ is definable from the parameters $\left(a_{n}\right)_{n<\omega},\left(M_{\beta}\right)_{\beta<\alpha}$, and $\sqsubset \cap M_{\alpha}$. Since these parameters are elements of $M_{\alpha+1}$, we have $\left(a_{\beta}\right)_{\beta<\alpha} \in M_{\alpha+1}$.

By (1) and (2)(i), $\left\{a_{\beta}: \beta<\omega_{1}\right\}$ is pairwise almost disjoint. To show that it is maximal, suppose that it were not. Then there is some $b \in[\omega]^{\aleph_{0}}$ such that $b$ is almost disjoint from all the $a_{\alpha}$ 's. Let $\left\{b_{n}: n \in \omega\right\} \subseteq \mathcal{P}(\omega) \cap M^{*}$ be a countable set generating $\left(\mathcal{P}(\omega) \cap M^{*}\right) \uparrow b$. Let $\alpha^{*}<\omega_{1}$ be such that $\left\{b_{n}: n \in \omega\right\} \subseteq M_{\alpha^{*}}$. Since $a_{\alpha^{*}}$ and $b$ are almost disjoint there is $n^{*} \in \omega$ such that $\left|b_{n^{*}} \cap a_{\alpha^{*}}\right|<\aleph_{0}$. By (2)(ii), there is a $u \in[\alpha]^{<\aleph_{0}}$ such that $\left|b_{n^{*}} \backslash \bigcup_{\beta \in u} a_{\beta}\right|<\aleph_{0}$. As $b \subseteq b_{n^{*}}$, it follows that $\left|b \backslash \bigcup_{\beta \in u} a_{\beta}\right|<\aleph_{0}$. But this is a contradiction to the choice of $b$.

Irreversibility of the implications in Lemma 8.11 cannot be proved in ZFC:

Lemma 8.13. Assume $\mathrm{CH}$. Then $\mathcal{M}_{\chi}=\mathcal{M}_{\chi}^{\sqsubset}$. In particular, for every partial order $P, \operatorname{SEP}(P)$ if and only if $\operatorname{SEP}^{+}(P)$.

Proof. $\mathcal{M}_{\bar{\chi}} \subseteq \mathcal{M}_{\chi}$ by Lemma 8.7. To show $\mathcal{M}_{\chi} \subseteq \mathcal{M}_{\chi}$, suppose $M \in \mathcal{M}_{\chi}$. Then by Lemma 3.4, $[M]^{\aleph_{0}} \subseteq M$. Let $\sqsubset$ be an arbitrary well-ordering of $M$ of order type $\omega_{1}$. Then, for every $x \in M, M_{\sqsubset x}$ and $\sqsubset \cap\left(M_{\sqsubset x}\right)^{2}$ are countable subsets of $M$ and hence, by $\mathrm{CH}$, elements of $M$. This shows that $M \in \mathcal{M}_{\chi}^{\sqsubset}$.

Even under $\neg \mathrm{CH}$, SEP and $\mathrm{SEP}^{+}$can be equivalent for partial orders with an " $\aleph_{2}$-version" of IDP.

Let us say that a partial order $P$ has the $\aleph_{2}$-IDP if for any sufficiently large $\chi$ and $M \preccurlyeq \mathcal{H}_{\chi}$, if $|M|=\aleph_{2}, P \in M$ and $[M]^{\aleph_{1}} \cap M$ is cofinal in $[M]^{\aleph_{1}}$ then $P \cap M \leq_{\aleph_{2}} P$ where $P \leq_{\aleph_{2}} Q$ is defined just as in Definition 4.1 with "countable" there replaced by "of cardinality $<\aleph_{2}$ ".

Note that every partial order of cardinality $\leq \aleph_{2}$ has the $\aleph_{2}$-IDP.

Theorem 8.14. Assume $\square_{\omega_{1}}$. For any partial order $P$ with the $\aleph_{2}$-IDP, $\operatorname{SEP}(P)$ if and only if $\mathrm{SEP}^{+}(P)$.

For the proof of Theorem 8.14 we use the fact that the class of partial orders with SEP is closed under $\leq{ }_{\sigma}$-suborders.

Lemma 8.15. For partial orders $P$ and $Q$, if $\operatorname{SEP}(P)$ and $Q \leq{ }_{\sigma} P$ then $\operatorname{SEP}(Q)$.

Proof. Fix a sufficiently large regular $\chi$. It is enough to show that, for every $M \preccurlyeq \mathcal{H}_{\chi}$ with $P, Q \in M$, if $P \cap M \leq_{\sigma} P$ then $Q \cap M \leq_{\sigma} Q$. To see this, let $x_{0} \in Q$ and we show that $Q \cap M\left\lceil x_{0}\right.$ has a countable cofinal subset. (That $Q \cap M \uparrow x_{0}$ has a countable coinitial subset can be proved similarly.) By $P \cap M \leq_{\sigma} P$ there is a countable set $X \subseteq(P \cap M)\left\lceil x_{0}\right.$ such that $X$ is cofinal in $(P \cap M)\left\lceil x_{0}\right.$. By $M \models Q \leq_{\sigma} P$ and elementarity, for every $x \in X$ 
we can find $X_{x} \in M$ such that $M \models$ " $X_{x}$ is cofinal in $Q\left\lceil x\right.$ ". Then $X_{x} \subseteq M$ and $X_{x}$ is a countable cofinal subset of $(Q \cap M)\left\lceil x\right.$. Letting $Y=\bigcup_{x \in X} X_{x}$, we have $Y \subseteq(Q \cap M)\left\lceil x_{0}\right.$ and $Y$ is countable. Moreover, $Y$ is a cofinal subset of $(Q \cap M)\left\lceil x_{0}\right.$ : If $y \in(Q \cap M)\left\lceil x_{0}\right.$, then in particular $y \in(P \cap M)\left\lceil x_{0}\right.$. Hence there is $x \in X$ such that $y \leq x$. Since $M \models y \in Q\left\lceil x\right.$, there is $x^{\prime} \in X_{x} \subseteq Y$ such that $y \leq x^{\prime}$.

Note that the proof above actually shows that each of the variants of SEP considered above (and also IDP and WFN) is closed under $\leq_{\sigma}$-suborders.

Proof of Theorem 8.14. If $|P|<\aleph_{2}$, then the assertion of the theorem is trivial. Hence we may assume $|P| \geq \aleph_{2}$. If $\operatorname{SEP}^{+}(P)$ then $\operatorname{SEP}(P)$ by Lemma 8.11(2). So we assume $\operatorname{SEP}(P)$ and prove $\mathrm{SEP}^{+}(P)$.

Let $\chi$ be sufficiently large and let $X$ be an arbitrary element of $\left[\mathcal{H}_{\chi}\right]^{\aleph_{1}}$. We show that there is $M \in \mathcal{M}_{\chi}$ such that $X \subseteq M$ and $P \cap M \leq_{\sigma} P$.

Fix a well-ordering $<^{*}$ of $\mathcal{H}_{\chi}$ of order type $\left|\mathcal{H}_{\chi}\right|$. Let $\mathcal{C}=\left\{C_{\alpha}\right.$ : $\left.\alpha \in \operatorname{Lim}\left(\omega_{2}\right)\right\}$ be a $\square_{\omega_{1}}$-sequence.

Let $\left(M_{\alpha}\right)_{\alpha<\omega_{2}}$ and $\left(a_{\alpha, \gamma}\right)_{\alpha<\omega_{2}, \gamma<\omega_{1}}$ be sequences defined inductively so that they satisfy the following conditions:

(0) $\left(M_{\alpha}\right)_{\alpha<\omega_{2}}$ is a continuously increasing sequence of elementary submodels of $\left(\mathcal{H}_{\chi}, \in,<^{*}\right)$ of cardinality $\aleph_{1}$.

(1) $\omega_{1}, X \subseteq M_{0}, P, \mathcal{C} \in M_{0}$.

(2) For all $\alpha<\omega_{2},\left(a_{\alpha, \gamma}\right)_{\gamma<\omega_{1}}$ is an enumeration of $M_{\alpha}$.

(3) For all $\beta<\omega_{2}$ we have $\left(M_{\alpha}\right)_{\alpha \leq \beta},<^{*} \cap\left(\bigcup_{\alpha \leq \beta} M_{\alpha}\right)^{2},\left(a_{\alpha, \gamma}\right)_{\alpha \leq \beta, \gamma<\omega_{1}}$ $\in M_{\beta+1}$.

(4) For all $\alpha<\omega_{2}, P \cap M_{\alpha+1} \leq_{\sigma} P$.

Let $M=\bigcup_{\alpha<\omega_{2}} M_{\alpha}$. Then $P \cap M \leq_{\aleph_{2}} P$ by the $\aleph_{2}$-IDP of $P$. From (4) it follows that $P \cap M \leq_{\sigma} P$. Hence by Lemma 8.15, $\operatorname{SEP}(P \cap M)$. It follows from Lemma 6.3 that there is $\alpha^{*} \in E_{\omega_{1}}^{\omega_{2}}$ such that $P \cap M_{\alpha^{*}} \leq_{\sigma} P \cap M \leq_{\sigma} P$. Since $X \subseteq M_{\alpha^{*}}$ by (1), the proof is complete if we can show the following:

Claim. $M_{\alpha^{*}} \in \mathcal{M}_{\bar{\chi}}$.

Let $C=C_{\alpha^{*}}$. Then $C$ is a cofinal subset of $\alpha^{*}$ of order type $\omega_{1}$. Let $\left(\xi_{\alpha}\right)_{\alpha<\omega_{1}}$ be strictly increasing enumeration of $C$. For each limit ordinal $\alpha<\omega_{1}$ there is $\beta<\alpha^{*}$ such that $\xi_{\alpha} \in M_{\beta}$. Since $C_{\xi_{\alpha}}=\left\{\xi_{\gamma}: \gamma<\alpha\right\}$ by coherence and since $C_{\xi_{\alpha}} \in M_{\beta}$, we have $\left\{\xi_{\gamma}: \gamma<\alpha\right\} \in M_{\beta} \subseteq M_{\alpha^{*}}$. Hence

$$
\left\{\xi_{\gamma}: \gamma<\alpha\right\} \in M_{\alpha^{*}} \quad \text { for all } \alpha<\omega_{1} .
$$

Let $\varphi: \omega_{1} \rightarrow \omega_{1} \times \omega_{1}, \alpha \mapsto\left(\varphi_{0}(\alpha), \varphi_{1}(\alpha)\right)$, be a surjection such that $\varphi \in M_{0}$. 
We now define a continuously increasing sequence $\left(N_{\alpha}\right)_{\alpha<\omega_{1}}$ of countable elementary submodels of $M_{\alpha^{*}}$ such that

(5) $a_{\xi_{\varphi_{0}(\alpha)}, \varphi_{1}(\alpha)},\left(N_{\beta}\right)_{\beta \leq \alpha} \in N_{\alpha+1}$ for all $\alpha<\omega_{1}$.

(6) $N_{\alpha+1}$ is the countable elementary submodel of $M_{\alpha^{*}}$ with $N_{\alpha+1} \in M_{\alpha^{*}}$ which is minimal with respect to $<^{*}$ satisfying (5).

That this construction is possible can be seen as follows: By $(*)$ and since the predicate " $N_{\alpha} \preccurlyeq M_{\alpha^{*}}$ " can be replaced by " $N_{\alpha} \preccurlyeq M_{\eta}$ " for a sufficiently large ordinal $\eta<\alpha^{*}$, each initial segment of $\left(N_{\alpha}\right)_{\alpha<\omega_{1}}$ is definable in $\mathcal{H}_{\chi}$ with parameters in $M_{\alpha^{*}}$ and hence is an element of $M_{\alpha^{*}}$.

By (5), $\bigcup_{\alpha<\omega_{1}} N_{\alpha}=M_{\alpha^{*}}$ and $\left(N_{\beta}\right)_{\beta \leq \alpha} \in N_{\alpha+1}$ for all $\alpha<\omega_{1}$. From Lemma 8.9 it follows that $M_{\alpha^{*}} \in \mathcal{M}_{\chi}$. This finishes the proof of the claim and hence of the theorem.

Corollary 8.16. Suppose that $\square_{\omega_{1}}$ holds and $\mathcal{P}(\omega)$ has the $\aleph_{2}$-IDP (in particular this is the case if $2^{\aleph_{0}}=\aleph_{2}$ ). Then $\operatorname{SEP}(\mathcal{P}(\omega)$ ) implies $\mathfrak{a}=\aleph_{1}$.

Proof. Under the assumptions, if $\operatorname{SEP}(\mathcal{P}(\omega))$ then we have $\mathrm{SEP}^{+}(\mathcal{P}(\omega))$ by Theorem 8.14 . Hence $\mathfrak{a}=\aleph_{1}$ by Theorem 8.12 .

9. Conclusion. As we have mentioned in the introduction, large cardinals are necessary to construct a Boolean algebra $A$ with the IDP but without the WFN. In this sense, IDP and WFN are pretty much the same and it is not surprising that all the interesting set-theoretic consequences of $\operatorname{WFN}(\mathcal{P}(\omega))$ that have been discovered so far already follow from $\operatorname{IDP}(\mathcal{P}(\omega))$. Looking at the proofs of the known consequences of $\operatorname{WFN}(\mathcal{P}(\omega))$ or $\operatorname{IDP}(\mathcal{P}(\omega))$, it turns out that most of the time $\operatorname{SEP}(\mathcal{P}(\omega))$ is enough to derive these consequences. An exception could be the equality $\mathfrak{a}=\aleph_{1}$, which is not known to follow from $\operatorname{SEP}(\mathcal{P}(\omega))$, but which follows from $\operatorname{IDP}(\mathcal{P}(\omega))$. The natural open question is whether $\operatorname{SEP}(\mathcal{P}(\omega))+\mathfrak{a}>\aleph_{1}$ is consistent.

One nice feature of $\operatorname{SEP}(\mathcal{P}(\omega))$ is that it holds in Cohen models. This does not have to be true for $\operatorname{WFN}(\mathcal{P}(\omega)$ ) (assuming large cardinals). We do not know about $\operatorname{IDP}(\mathcal{P}(\omega))$. As it turns out, $\operatorname{SEP}(\mathcal{P}(\omega))$ is relatively robust under slight changes of the definition. It does not matter whether we demand the existence of a single elementary submodel of $\mathcal{H}_{\chi}$ with certain properties, or of stationarily many, or of cofinally many. Therefore it is interesting to know that the strongest variant of SEP along these lines, IDP, is strictly stronger than SEP. In some sense, we get the best possible result here. There is (in ZFC) a Boolean algebra with SEP but without IDP and it is consistent that $\mathcal{P}(\omega)$ itself is an example. 


\section{References}

[1] A. Blass, Combinatorial cardinal characteristics of the continuum, in: Handbook of Set Theory, to appear.

[2] A. Dow and K. P. Hart, Applications of another characterization of $\beta \mathbb{N} \backslash \mathbb{N}$, Topology Appl. 122 (2002), 105-133.

[3] A. Dow and P. Simon, Thin-tall Boolean algebras and their automorphism groups, Algebra Universalis 29 (1992), 211-226.

[4] M. Foreman, M. Magidor and S. Shelah, Martin's Maximum, saturated ideals, and non-regular ultrafilters. Part I, Ann. of Math. 127 (1988), 1-47.

[5] S. Fuchino, S. Geschke, S. Shelah and L. Soukup, On the weak Freese-Nation property of complete Boolean algebras, Ann. Pure Appl. Logic 110 (2001), 89-105.

[6] S. Fuchino, S. Geschke and S. Soukup, The weak Freese-Nation property of $\mathcal{P}(\omega)$, Arch. Math. Logic 40 (2001), 425-435.

[7] S. Fuchino, S. Koppelberg and S. Shelah, Partial orderings with the weak FreeseNation property, Ann. Pure Appl. Logic 80 (1996), 35-54.

[8] S. Fuchino and L. Soukup, More set theory around the weak Freese-Nation property, Fund. Math. 154 (1997), 159-176.

[9] I. Juhász and K. Kunen, The power set of $\omega$. Elementary submodels and weakenings of $C H$, ibid. 170 (2001), 257-265.

[10] I. Juhász, L. Soukup and Z. Szentmiklóssy, Combinatorial principles from adding Cohen reals, in: Logic Colloquium '95 (Haifa), Lecture Notes in Logic 11, Springer, 1998, 79-103.

[11] - - - - , What is left of CH after you add Cohen reals?, Topology Appl. 85 (1998), $165-174$.

[12] W. Just, A modification of Shelah's oracle-c.c. with applications, Trans. Amer. Math. Soc. 329 (1992), 325-356.

[13] S. Shelah, Proper and Improper Forcing, 2nd ed., Perspect. Math. Logic, Springer, 1998.

[14] S. Shelah and J. Steprāns, Non-trivial automorphisms of $\beta N \backslash N$ without the continuum hypothesis, Fund. Math. 132 (1989), 135-141.

Department of Natural Science and Mathematics

College of Engineering

Chubu University

Kasugai, Aichi 487-8501, Japan

E-mail: fuchino@isc.chubu.ac.jp
II. Mathematisches Institut Freie Universität Berlin Arnimallee 3 14195 Berlin, Germany E-mail: geschke@math.fu-berlin.de

Received 18 September 2003;

in revised form 17 March 2004 